ENERGY MANAGEMENT FOR A MULTIPLE-PULSE MISSILE

by

Craig Alan Phillips

Thesis submitted to the Graduate Faculty of the Virginia Polytechnic Institute and State University in partial fulfillment of the requirements for the degree of

\author{
MASTER OF SCIENCE \\ in \\ Aerospace and Ocean Engineering
}

Approved:

Dr. E.M. Cliff] Chairman

Dr. H.J. Kellev

September, 1986

Blacksburg, Virginia 


\title{
ENERGY MANAGENENT FOR A MULTIPLE-PULSE MISSILE
}

\author{
by \\ Craig Alan Phillips \\ Committee Chairman: Eugene M. Cliff \\ Aerospace and Ocean Engineering \\ (ABSTRACT)
}

A nonlinear programming technique is applied to the optimization of the thrust and lift control histories for missiles. The first problem considered is that of determining the thrust history which maximizes the range of a continuously-variable (non-pulsed) thrust rocket in horizontal lifting flight. The optimal control solution for this problem is developed. The problem is then approximated by a parameter optimization problem which is solved using a second-order, quasi-llewton method with constraint projection. The two solutions are found to compare well. This result allows confidence in the use of the nonlinear-programming technique to solve optimization problems in flight mechanics for which no analytical optimal-control solutions exist. Such a problem is to determine the thrust and lift histories which maximize the final velocity of a multiple-pulse missile. This problem is solved for both horizontal- and elevation-plane trajectories with and without final time constraints. The method is found to perform well in the solution of these optimization problems and to yield substantial improvements in performance over the nominal trajectories. 


\section{ACKNOUILEDGEIIENTS}

The author wishes to express his appreciation to Dr. Eugene Cliff, his Research Advisor, and to Dr. H.J. Kelley and Dr. Frederick Lutze for the direction which they provided during the completion of this study. Also, the efforts of Mr. M.E. Packard and Uday Shankar in the movement of the required software to the Naval Surface Heapons Center is greatly appreciated. 
TABLE OF CONTENTS

\section{$\underline{\text { Page }}$}

Abstract........................... $i i i$

Glossary......................... v v

Chapter 1. Introduction....................... 1

Chapter 2. Discussion of Methodology.............. 5

Chapter 3. Continuously-Variable-Thrust

Lifting-Flight Optimization............ 12

3.1 Overview....................... 12

3.2 Analytical Solution.............. 12

3.3 Numerical Optimization of the Continuous ly-Variable-Thrust

Problem...........................

3.4 Comparison of Analytical and Numerical Results................. 28

Chapter 4. Pulsed-Motor Trajectory Optimization...... 36

4.1 Overview...................... 36

4.2 Parameterization................. 37

4.3 Trajectory Mode 1................ 42

4.4 Horizontal-Pulsed Flight.......... 45

4.5 Elevation-Plane Trajectories........ 57

Chapter 5. Conclusions........................ 75

References......................... 81

Appendix A Missile Airframe Characteristics

and Atmosphere Model.................. 82 


\section{LIST OF TABLES}

Table

Page

3.1

Missile Characteristics

13

3.2

Initial Conditions for the ContinuouslyVariable Thrust Horizontal Flight

Problem

Final Conditions for the ContinuouslyVariable Thrust Horizontal-Flight

Problem

4.1 Initial and Final Constraints for Horizontal Pulsed Flight ..............

Two-Pulse Horizontal-Flight Inequality

Constraints

Three-Pulse Horizontal-Flight

Inequality Constraints

Parameter Values for Optimal HorizontalPulsed Flight .......................

Final Values for the Optimal HorizontalPulsed Flight

Initial Conditions for Elevation-Plane

Trajectories

Final Conditions for Elevation-Plane Trajectories

Nominal Two-Pulse Elevation-Plane Problem Constraints

4.9 Elevation-Plane Optimization Parameter

Optimal Single and Double Pulse Elevation Plane Trajectory Final Values .......... 


\section{LIST OF TABLES (Continued)}

Table

Page

A. 1

Aerodynamic Drag Coefficients for

Missile Airframe

83

A.2

Maximum Airframe Lift Coefficients

85

A. 3

Missile Weight Breakdown

86

A. 4

Missile Propulsion Characteristics

87

A. 5

Atmospheric Model...............

89 


\section{LIST OF FIGURES}

Figure

Page

3.1 Singular Arc for the Horizontal

Flight Problem ....................... 19

3.2 Typical Mass Flow Rate History .......... 26

3.3 Optimal Control and Parameter

Optimization Trajectories ...............

3.4 Optimal Control and Parameter Optimization Mass Flow Rate Histories for One Segment Sustain Rate Spline ..................

3.5 Optimal Control and Parameter Optimization Mass Flow Rate Histories for Two Segment Sustain Phase Spline ................. 35

4.1 Burn Rate History for Three Pulse Motor ... 38

4.2 Typical Load Factor History ............ 41

4.3 One-Pulse Horizontal Flight Velocity

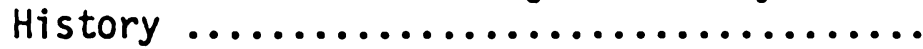

4.4 Two-Pulse Horizontal Flight Velocity

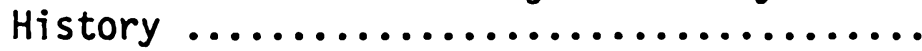

4.5 Three-Pulse Horizontal Flight Velocity

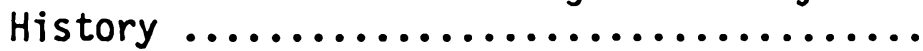

4.6 Elevation Plane One- and Two-Pulse Trajectory Shapes ....................

4.7 Elevation Plane One- and Two-Pulse Velocity Histories ....................

4.8 Effect of Time of Flight Constraint on Terminal Conditions 


\section{GLOSSARY OF SYPABOLS}

$A_{e}$

The area of the rocket nozzle exhaust plane

$\mathrm{C}_{\mathrm{DO}}$

The zero angle of attack drag coefficient

$C_{L}$

The lift coefficient

D

The aerodynamic drag

The specific energy

$E_{2}$

The integral square penalty on the lift coefficient

g

The acceleration due to gravity, 32.174 feet/second

H

The pre-Hamiltonian for the optimal control problem

h

The altitude in feet

J

The performance index for the optimal control problem

k

The induced drag factor

m

The mass of the missile

Pa

The ambient atmospheric pressure

P

The rocket motor exhaust pressure

S

The aerodynamic reference area or body cross-sectional area

T The missile thrust

$t$ The independent variable for the optimal control problem or time

u

The control or the rocket motor burn rate

$u^{* *}$

The optimal control history

$V \quad$ The missile speed

$V_{e} \quad$ The exhaust velocity of the rocket motor 
The missile weight

$x \quad$ The state vector for the optimal control problem or the missile downrange coordinate

$x^{*} \quad$ The state vector history produced by the optimal control

Greek Symbols

B Mass flow rate

$\gamma \quad$ Flight path angle

$\theta \quad$ End manifold for optimal-control problem

$\zeta \quad$ Atmosphere density 


\section{Chapter 1}

\section{Introduction}

One of the most important aspects in the design of flight vehicles is the selection of flight profiles which yield the greatest performance. This selection of the profile plays a substantial role in the preliminary design of flight veriicles in that in the evaluation of competing configurations the use of an ad-hoc profile or control policy may penalize the performance of one design more heavily than another. Thus, to guarantee the selection of the best design it is important to optimize the profile and related control policies for each configuration early in the design process. For tactical missiles, the flight profiles are determined by the thrust and load-factor histories. l!hich flight profiles are best is a function of many aspects of the design including the geometry of the missile, whether the missile is propelled by liquid or solid fuel, the performance which the designer wishes to maximize, and the constraints upon its operation. The designer may wish to optimize any one of a number of traditional missile-performance factors such as maximum velocity at a given point, minimum time to a given point, maximum altitude, or maximum downrange for some fixed time interval. Also, the designer is forced to impose constraints on the proposed system to allow proper operation of missile subsystems or to perform mission objectives. An example of the latter would be the specification of a final altitude and range to allow interception of the target. Each combination of missile factors and desirable missile 
performance will result in a new set of "best" or optimal thrust and load-factor histories. The procedure for determining these values is difficult at best. Analytical solutions are available for only a very few problems since the complexity of the solution increases extremely rapidly with added complexity in the initial problem definition. Analytical solutions require that extremely simplified models of the missile dynamics be used along with simple performance requirements. Thus most realistic problems lie outside the scope of these analytical results and require some type of numerical procedure for their solution.

The present study seeks to solve three problems of reasonable complexity; one of these has an analytical solution, while the other two do not. The first problem is that of maximizing the total range of a continuously-variable thrust solid-fuel rocket in one-dimensional. horizontal, lifting flight. For vehicles without Mach-number dependence in their aerodynamic characteristics, the extremal mass-velocity scheduling was determined by Hibbs (1). Miele (2) was able to demonstrate that the Hibbs solution met the sufficiency conditions for an optimum solution and was also able to determine the solution for vehicles with Mach-number-dependent aerodynamic characteristics. In the present study, the analytical solution will be presented for the vehicle without Mach-number dependency in the aerodynamic characteristics as yill be the corresponding parameter-optimization solution.

The second problem considered will be that of maximizing the final velocity of a single-stage missile with a pulsed, solid motor at a specified final altitude and range. This problem is of significant 
practical interest in that many modern fire-control systems designate a target point to which the missile mid-course guidance must fly the missile and at which the terminal homing guidance must take over to intercept the target. Maximizing the velocity at this handover to the terminal guidance will enhance the probability of intercepting the target. Both the problem of horizontal flight and elevation-plane flight, which correspond to air-launched and surface-launched missiles, respectively, are considered. The missile characteristics available for optimization include the load-factor history, the fuel allocation bet:veen the pulses and the ignition times of each of the pulses. Variations in the motor mass-flow rate during operation result in significant degradation in delivered specific impulse and added design complexity. Thus, the constant mass-flow rate, pulsed motor offers significant advantages in the practical design of rocket motors and will be the motor configuration used for this study. The mass-flow rate of the motor will be considered fixed to reflect the non-performance constraints placed on operational systems. These constraints include launcher-mass-flow-rate limits or nozzle/airframe packaging limits. The optimal pulsing and load factor histories for the final-time-free and the time-constrained scenario will be considered.

A third problem considered is that of determining the minimum-time-of-flight elevation-plane trajectory for a pulsed. solid-fuel, single-stage missile to a specified final altitude and range. 
No analytical solution is available for any of these pulsed rocket motor problems; but the optimal-control problem will be approximated by a parameter-optimization problem and solved by a numerical procedure utilizing a quasi-Newton methodology.

Chapter two will present an overview of the methodologies available to solve optimal-trajectory problems. Chapter three presents the continuously-variable thrust programming analysis and results. Chapter four will present the pulsed-motor details and results. 


\section{Chapter 2}

\section{Discussion of the Methodology}

This chapter presents the approaches available to determine the best control histories for problems in flight mechanics. These techniques fall into two main categories. The first of these is the optimal-control theory methodology which allows solution either numerically or analytically. The second main area involves an approximation to the optimal-control problem through the use of parameter optimization. Such parameterized problems may be solved by numerical procedures. Both problem formulations are presented along with the relationship between the methodologies and the procedures available for their solution.

Before considering a trajectory-optimization problem one may define a (somewhat) general optimal-control problem. A primary ingredient of the problem is the description of the system dynamics by a set of differential equations in first-order form.

$$
\frac{d x(t)}{d t}=\rho(x(t), u(t))
$$

Here $x(t)$ is an $n$-dimensional state vector and $u(t)$ is a m-dimensional control vector. In addition to the dynamical equations (2.1a) one has the boundary conditions:

$$
x\left(b_{0}\right)=x_{0}
$$




$$
\Phi\left(x\left(t_{1}\right), t_{1}\right)=0
$$

In (2.1b) $t_{0}$ is a specified initial time, and $x_{0}$ is a specified initial vector. Since the differential system $(2.1 \mathrm{a})$ is autonomous, one may take the initial time $\left(t_{0}\right)$ to be zero. The function $\Phi$ in (2.1c) may be vector-valued; one thinks of this constraint as defining a terminal manifold. The condition (2.1b) can be generalized; however, the stated form is sufficient for the applications here. In many applications the control $u(t)$ must lie within prescribed bounds; mathematically one has:

$$
U_{\min } \leq u(t) \leq U_{\max }
$$

Where $U_{\min }$ and $U_{\max }$ are fixed m-vector bounds. Again, more general forms of constraint are possible.

The final ingredient of an optimal-control problem is a "payoff" function which describes the quantity to be maximized. For the present purposes this can be written as

$$
J(u)=\int_{0}^{t_{1}} L(x(t), u(t)) d t
$$

The optimal-contol problem is to find a function $u^{*}$, satisfying the constraint (2.1d), such that the solution to (2.1a) with initial data (2.1b) reaches the terminal manifold (given by (2.1c)), and such that 
the payoff function is maximal. That is, any other control satisfying (2.1) produces a value of $\mathrm{J}$ which is no greater than $\mathrm{J}\left(\mathrm{u}^{*}\right)$.

In flight-mechanics applications many optimal-control problems are quite challenging. In order to "solve" such problems the analyst can employ the Maximum Principle (see, for example, the text by Leitmann $(3))$. For the control, $u^{*}(t)$, to be an optimal control it must satisfy the Maximum Principle as given by Leitmann. This principle is conveniently stated in terms of a pre-Hamitonian function defined by:

$$
\left.H(\lambda, x, u)=\lambda_{0} L(x, u)+\lambda^{T}(t) / x, u\right)
$$

The Maximum Principle (3) states that if $u^{*}(t), t_{0}<t<t_{1}$, is an optimal control then there exists a non-zero continuous vector function, $\lambda$. which is solution of the adjoint equations:

$$
\frac{d \lambda_{l}}{d t}=-\frac{\partial H}{\partial x_{l}} \quad i=1, \ldots, n
$$

where $u=u^{*}(t), x=x^{*}(t)$, and $\lambda=\lambda(t)$ for $t_{0}<t<t_{1}$.

The optimal control will meet the following conditions.

(1) $\max H(\lambda(t), x(t), u)=H\left(\lambda(t), x^{*}(t), u^{*}(t)\right)$

(2) $H\left(\lambda(t), x^{*}(t), u^{*}(t)\right)=0$

(3) $\lambda_{0}(t)=$ constant $\leq 0$ 
(4) The vector $\left(\lambda_{1}(t), \lambda_{2}(t), \ldots \ldots, \lambda_{n}(t)\right)$ is normal to the end
manifold $\theta_{1}$ at $t=t_{1}$ ?

It should be noted that the Maximum Principle is a necessary condition for the optimality of a control, for it states the conditions which must be satisfied for a control to be optimal. A control satisfying the necessary conditions of the Maximum Principle is an extremal control which may or may not be an optimal control; i.e. the Maximum Principle is not sufficient. Thus, an optimal control is extremal; but an extremal control is not necessarily optimal. The Maximum Principle provides a means of constructing candidates for optimal control. If one or more optimal controls exists (uniqueness is not guaranteed) then it furnishes a maximum of the cost function. The procedure for determining the optimal control is to establish the existence of an optimal control for the problem, if possible, and to determine all of the extremal controls. Among this candidate set of extremal controls will be the optimal control. For most problems, the proof of the existence of the optimal control is difficult and one must resort to determining the best of the extremal controls and using knowledge of the problem to argue that this is indeed the optimal control. Thus the determination of the extremal controls requires the solution of a two-point boundary value problem composed of Equations 2.1 and 2.4. Analytical solutions are available for only the simplest of problems. For systems of even modest dimension some type of numerical solution is required. 
Mumerous numerical techniques for such problems are available and sometimes perform relatively well. An example of one such method is given in (4). But this technique presents difficulties in the operational environment. Since the adjoint equations (Equation 2.4) must be developed in a functionalized form, any change in the numerical description of the system requires that the analyst rework all of the representations. A small change in the aerodynamic description of the missile may require a great deal of work before the new extremal control solution could be generated by these codes. Not only do these codes have the disadvantage of the requirement of a significant volume of work for each solution but they also have the associated problem of the possibility of the inclusion of new errors with each new version of the problem. Since these methods work in an iterative fashion, an additional problem may be that the system under study may show extreme sensitivity to small perturbations in the boundary conditions and thus suffer instability. To solve such problems, the analyst must have a good knowledge of both the methodology and of the system. In a production environment these methods exhibit lengthy turn-around times along with quality-control problems. A more productive method would be one which is robust in its use, easily allows small changes in the missile description, and requires less knowledge of the methodology on the part of the analyst. Such a method is the topic of this study.

In the present study the optimal-control problem is approximated by a parameter-optimization problem. The use of parameter optimization for the determination of optimal control for flight-mechanics problems was 
demonstrated by !ell (5). In one approach to this parameterization, each of the elements of the $m$ dimensional control vector, $u(t)$, which represent continuous histories of each control with respect to the chosen independent variable are approximated by a series of linear splines joined at nodes. The values of the controls at each of these nodes become the parameters to be optimized. Thus if the number of nodes is $k$ then the control vector, $u(t)$, is replaced by the control-parameter vector, $u^{\prime}$, of dimension $k \cdot m$. In parameter optimization, the parameter vector, $u^{\prime}$, is sought which maximizes the performance index defined as a scalar function of the $\mathrm{k} \cdot \mathrm{m}$ control parameters, (i.e. $J^{\prime}\left(u^{\prime}\right)$ ) as follows. Given the $k \cdot m$ vector $u^{\prime}$ the corresponding control history $u(t)$ can be reconstructed. In principle, the differential equations (2.1a) can be "solved" from the given initial-value forward. One of the components of the end conditions (2.1c) is selected as a "stopping" condition and the state-vector $x(t)$ is propagated to this point. The performance integral (2.2) can be evaluated and this is the value assigned to $\mathrm{J}$ '. The unused end condition (2.1c) and the control constraints (2.1d) define a vector-valued constraint function $g\left(u^{\prime}\right) \leq 0$. In paraneter-approximation the problem is to find the $k \cdot m$ vector $u^{\prime}$ which maximizes $J '$ among all such vectors which satisfy the constraints, $g\left(u^{\prime}\right) \leq 0$. Thus the optimal parameter solution will only approximate the true optimal-control solution and will be suboptimal. As the grid of nodes points of the linear splines become finer the parameter solution will approach the optimal solution. 
Parameterization schemes may be used to characterize the control other than the node values of the control history splines. For the present study, the load factor history is represented by these nocial values but the thrust history is characterized by other parameterizations which utilize parameters unique to pulse motors. Constraints on the states or controls which must be continually enforced during the trajectory may be handled by means of an integral-square penalty as described by Kelley (6). An example would be that the lift coefficient may not exceed some specified maximum value during the flight.

As with optimal control problem, many numerical procedures exist to solve parameter optimization problems. The simplest procedures are the gradient or "steepest-descent" techniques. Second-order methods are also available to improve the rate of convergence. One such method is Newton-Raphson iteration which requires the evaluation of the Hessian matrix. Quasi-Newton or variable-metric methods avoid the evaluation of the Hessian matrix by using iterative evaluation of first order information to approximate the Hessian matrix. One such quasi-Hewton method. the Broyden-Fletcher-Goldfarb-Shanno (BFGS) procedure is implemented with constraint projection as described in (7). 


\section{Chapter 3}

Continuous ly-Variable-Thrust Lifting-Flight Optimization

\subsection{Overview}

One-dimensional maximum-range flight in the horizontal plane was one of the first optimal-trajectory problems successfully handled. This problem was first addressed by Hibbs (1); Miele (2) later employed a Green's function method to verify that the Hibbs solution was maximizing. Analytical solutions are available for mass-varying vehicles with and without Mach-dependent aerodynamic characteristics. In this chapter the optimal-control solution for a vehicle with Mach-independent aerodynamics will be developed and compared to the parameter-optimization solution. The analytical problem considered here is to determine the thrust program which will maximize the range of the missile while transferring it between given values of velocity and mass. The variations in the respective solutions will be discussed along with the implications for trajectory optimization.

\subsection{Analytical Solution}

The airframe characteristics used in this study are typical of those associated with modern solid-fuel missiles. The required missile parameters are given in Table 3.1. Propulsion for the missile is provided by a state-of-the-art solid-rocket motor with a sea-level specific impluse of 220.0 seconds. In the analytical treatment the 
TABLE 3.1

Missile Characteristics

Quantity

Initial Weight

Burnout lieight

Cross-section Area

Zero-Lift Drag

Coefficient

Induced Drag

Factor

Exhaust Velocity
Value

976.0 pounds

545.0 pounds

0.545 sq. ft.

0.1

0.079

$7100.0 \mathrm{ft} . / \mathrm{sec}$. 
aerodynamic characteristics will be considered independent of Hach number and a quadratic drag law will be assumed. The drag will be given by:

$$
D=\frac{1}{2} C_{D 0} \rho S V^{2}+\frac{2 k W^{2}}{\rho S V^{2}}
$$

The values of the zero-lift drag coefficient, $C_{D D}$, and the induced-drag factor, $k_{,}$are given in Table 3.1. The level-flight requirement of the problem constrains the lift to equal the missile weight at all points along the trajectory. The development of the governing equations of motion assumes the vehicle pitch dynamics to be fast enough to be separable and thus negligible.

Four physical variables, mass, velocity, time, and range, are associated with this problem. These may be nondimensionalized by the following relations.

$$
\begin{aligned}
& \bar{m}=\frac{2 m g}{V_{e}^{2} \rho S \sqrt{C_{D 0} / k}} \\
& \bar{V}=\frac{V}{V_{e}} \\
& \bar{x}=\frac{x g}{V_{e}^{2} \sqrt{C_{D 0^{k}}}} \\
& \bar{i}=\frac{t g \sqrt{C_{D 0} k}}{V_{e}}
\end{aligned}
$$


Throughout the remainder of the chapter these four quantities will be presented in their nondimensionalized form; thus the superscribed-bar notation will be dropped.

The equations of motion in nondimensional form are given by the following relations.

$$
\begin{gathered}
\frac{d V}{d t}=-\frac{1 d m}{m d t}-\frac{V^{2}}{m}-\frac{m}{V^{2}} \\
\frac{d m}{d t}=-\beta
\end{gathered}
$$

The only physical control, mass-flow rate, takes on the form $\beta$ in these equations.

While this problem was originally studied by Hibbs (1) for a vehicle with unbounded thrust, a more modern approach allows treatment of the bounded-thrust vehicle and provides greater insight into the solution. Following Leitmann (3), the problem may be defined precisely as determining the optimal control $\beta^{*}, t_{0}<t<t_{1}$ which transfers the system (3.3) from the initial state $\left(V_{0}, m_{0}\right)$ at $t=t_{0}$ to the final state $\left(V_{1}, m_{1}\right)$ at $t=t_{1}$ which maximizes the cost function (in Lagrange form):

$$
J=\int_{t_{0}}^{t_{1}} V d t
$$


For $\beta^{*}$ to represent an extremum control, then the necessary conditions of the l:aximum Principle as given by Leitmann (3) and presented in Chapter 2 must be satisfied.

Adjoining the equations of motion (Equation 3.3) to the integrand of the cost function (Equation 3.4) yields the pre-Hamilitonian in the following form:

$$
H=\lambda_{0} V-\lambda_{1}\left(\frac{V^{2}}{m}+\frac{m}{V^{2}}\right)+\beta\left(\frac{\lambda_{1}}{m}-\lambda_{2}\right)
$$

The adjoint equations are:

$$
\begin{aligned}
& \frac{d \lambda_{1}}{d t}=-\lambda_{0}+\lambda_{1}\left(2 \frac{V}{m}-2 \frac{m}{V^{3}}\right) \\
& \frac{d \lambda_{2}}{d l}=\lambda_{1}\left(-\frac{V^{2}}{m^{2}}+\frac{1}{V^{2}}+\frac{\beta}{m^{2}}\right)
\end{aligned}
$$

Where $\lambda_{0}$ is a non-positive scalar constant.

If the control history, $\beta(t)$ is to be optimal, it is necessary that at each instant the Hamiltonian, $H$, be maximized (as a function of the control, $\beta$ ). From the form of the Hamiltonian function (3.5), it is readily seen that the behavior of $\beta$ is dictated by the "switching function":

$$
\sigma=\frac{\lambda_{1}}{m}-\lambda_{2}
$$


Specifically, one should choose $\beta^{*}$ according to:

$$
\begin{aligned}
& \sigma(t)>0 \rightarrow \beta^{*}=\beta_{\max } \\
& \sigma(t)<0 \rightarrow \beta^{*}=\beta_{\min }
\end{aligned}
$$

A third possibility is that the control may be singular. This is a control history which produces a zero value of the switching function for a finite amount of time. This implies that the time derivative of the switching function also vanishes. This derivative is given by:

$$
\frac{d \sigma}{d t}=\frac{\dot{\lambda}_{1}}{m}+\frac{\lambda_{1} \beta}{m^{2}}-\dot{\lambda}_{2}
$$

In addition to the differential equations (3.3), (3.6) and the control specification (3.8), the Maximum Principle implies certain boundary conditions. Since the staes $V$ (velocity) and m (mass) are specified both at the initial and final points the "transversality" conditions yield no information about the boundary values for the adjoints $\lambda_{1}, \lambda_{2}$. However, since the final time is free, one has the associated transversality condition on $H(t)$. Moreover, since time does not appear explicitly in either the system equations (3.3) or in the cost function (3.4) then the Hamiltonian is constant; i.e.

$$
H(\lambda, V, m, t)=0
$$


Combining equations (3.6), (3.7), (3.9), and (3.10), it can be shown that:

$$
\frac{d \sigma}{d l}=0=\lambda_{1} W(V, m)
$$

where:

$$
W(V, m)=\frac{3}{V^{5}}+\frac{1}{V^{4}}-\frac{1}{m^{2} V}-\frac{1}{m^{2}}
$$

From equation (3.11), it can be seen that $\dot{\sigma}$ will vanish if either $\lambda_{1}=0$ or $W(V, m)=0$. At the singular arc $(\sigma=0)$ the first possibility requires $\left(\operatorname{see}(3.7)\right.$ and $(3.11)$ ) that $\lambda_{0}=\lambda_{1}(t)=\lambda_{2}(t)=0$. Since the Maximum Principle guarantees a non-zero adjoint vector if the control is extremal, this possibility can be excluded. From Equation (3.12), the equation for the singular arc is:

$$
m^{2}=V^{4} \frac{(V+1)}{(V+3)}
$$

The curve! described by Equation (3.13) divides the quarter plane of the velocity-mass parameter space $V>0$ and $m>0$ into regions denoted by I and II as shown on Figure 3.1. The derivative of the switching function is negative in region I, positive in region II, and zero along the singular arc. The switching function is continuous and thus it must pass through zero as it switches sign. Thus there may be a maximum of one switch in each region. As the switching function changes sign the 


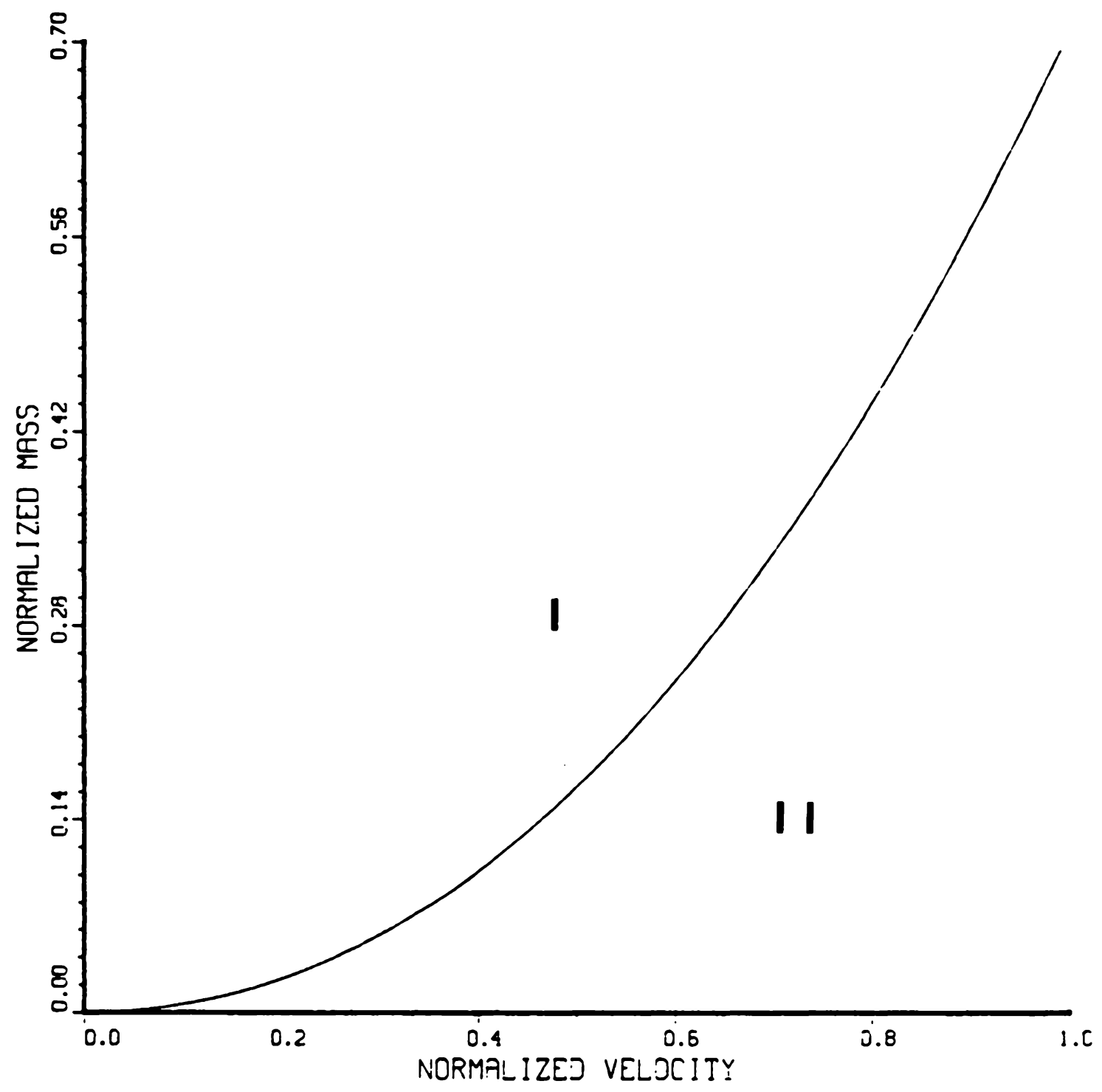

Figure 3.1 Singular Arc for the Horizontal Flight Problem 
associated extremal control, $\beta$ * switches according to (3.8). The construction of the optimal-control path through the velocity-mass space is dependent upon the initial and final states specified for the missile and the constraints placed upon the control, $\beta$. The optimal control for the unbounded-thrust missile will be developed first and will be followed by that for the bounded-thrust missile.

Unbounded-Thrust Missile

The unbounded-thrust missile is one for which the thrust may vary between an impulsive burn and a zero burn rate during the interval between ignition and fuel exhaustion. That is:

$$
\begin{aligned}
& \beta \min =0 \\
& \beta \max =\infty
\end{aligned}
$$

This implies that the rocket motor is "infinitely throttleable". It may be reignited any number of times until fuel exhaustion. There are four types of optimal control for this problem dependent upon the boundary conditions. Each of these will be discussed in detail in the text which follows. 


\section{Case I}

In this first case, the initial state of the missile is such that it lies in region I of the velocity-space shown in Figure 3.1 and the specified final state lies in region $I$. The missile starts at some

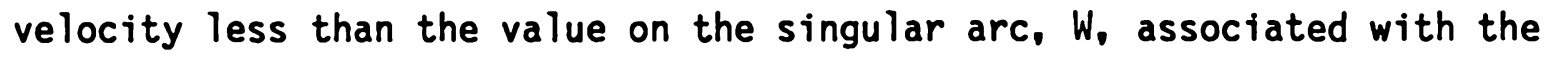
initial vehicle mass. The final velocity is also less than the singular-arc value for the missile burnout mass. At the initial point the switching function will be positive and the optimal arc for this case would consist of an initial impulsive burn until the switching function vanishes. If the fuel fraction of the missile is sufficiently large then the vanishing of the switching function denotes the intersection of this impulsive burn with the singular arc, W. At this point the mass-flow rate, $\beta$, would be throttled down to some intermediate value which would allow the singular arc to be followed until fuel exhaustion. Upon reaching burnout weight, the maximizing trajectory would depart the singular arc and the switching function would achieve a negative value. Thus, the remainder of the maximizing trajectory would consist of a zero-thrust glide until the final velocity boundary condition is achieved.

For the case where sufficient fuel fraction does not exist to allow the singular arc to be reached, the maximizing trajectory will consist of an impulse burn until fuel exhaustion at a point in region I of the velocity-mass space. It should be noted that throughout this trajectory the derivative of the switching function will be negative and that the 
switching function will switch from positive to negative at the moment of fuel exhaustion.

\section{Case II}

For this case the initial point lies in region I of the velocity-mass space and the final point in region II. The initial velocity of the missile is less than the singular-arc velocity associated with the initial mass of the vehicle. To satisfy the boundary conditions of this problem, the missile must be transferred from this initial velocity to a final velocity greater than the singular-arc value associated with the burnout mass of the missile. At the initial point the switching function is positive and the maximizing control consists of an initial impulsive burn until the singular are is reached and the switching function vanishes. A mass-flow rate schedule is then used which allows the singular arc to be followed until the maximizing trajectory must leave it to satisfy the boundary conditions. The final arc of the maximizing trajectory will consist of a second impulsive burn until the burnout mass and the specified final velocity are achieved.

\section{Case III}

The initial point in this case lies in region II of the velocity-mass space and the final point in region I. The initial velocity of the missile is greater than the singular-arc velocity associated with the initial mass of the missile. The final velocity of 
the missile is less than the singular-arc value for the final mass of the missile. The missile must be transferred between these two states. At the initial point the switching function is negative and the initial segment of the extremal trajectory consists of a zero thrust glide the until intersection with the singular arc. At this point the switching function vanishes and a mass-flow rate schedule of intermediate values is followed to stay on the singular arc until fuel exhaustion. Since the final mass value has been reached the maximizing trajectory must depart from the singular arc into Region I. At this point, the final arc of the maximizing trajectory consists of a zero-thrust glide to the final specified velocity.

\section{Case IV}

This final case for the unbounded-thrust missile involves an initial point which lies in region II of the velocity-mass space and the final point also in this same region but at the burnout mass. The missile must begin and end its trajectory at velocities greater than the singular-arc velocities associated with the initial and final mass values of the missile. At the initial point the switching function is negative and thus the initial arc consists of zero-thrust glide until intersection with the singular arc and the vanishing of the switching function. A mass-flow rate schedule of intermediate values is then followed to stay on the singular-arc. At a point along the singular arc the maximizing trajectory must depart the singular arc in order to 
satisfy the boundary conditions. As in Case II, this occurs at a point in the trajectory such that an impulsive burn will allow all of the remaining fuel to be burned just as the specified final velocity is reached.

\section{Bounded-Thrust Missile}

The bounded-thrust missile is one for which there is a finite upper limit and (possibly) a positive lower limit on the allowable mass-flow rate. The engine is not re-ignitable; once shut off it can not be restarted which is reflected in the analytical optimal control problem by the lower limit placed on the mass-flow rate. Of course, after fuel burnout the thrust will be zero. For the bounded-thrust missile, singular control may not be possible. If the mass-flow rate to follow the singular arc violates either the upper or the lower bound then it will be impossible to follow the solutions outlined for the unbounded missile. This is the distinguishing characteristic of the bounded-thrust problem. For a typical missile application with a low-drag profile, the singular-arc mass-flow rate schedule is most likely to violate the lower mass-flow-rate bound associated with realistic rocket motor operation. For missiles where singular control is possible, then the optimal solution will be the same as for the unbounded thrust missile except that the impulsive-burn arc will be replaced by a maximum-burn-rate arc. 
3.3 Numerical Optimization of the Continuously-Variable Thrust Problem Since the missile load-factor history is specified by the level flight requirement, the only missile characteristics available for optimization are those associated with the solid-fuel motor. The problem considered will be equivalent to that of Case I of the analytical solution but with an upper bound on the mass-flow rate. Thus, a maximum-mass-flow rate arc will be substituted for the initial impulsive arc. Knowledge of the optimal-control solution is used as a guide to the design of the parameterization which consists of three parameters available for optimization. The first parameter represents the fuel fraction to be used in the initial, maximum-thrust burn. Ideally, this fuel fraction should be the fraction which delivers the missile onto the singular arc. For the remainder of the thrusting arc, the mass flow-rate is represented by a one-segment linear spline with the fraction of total fuel-mass consumed as the independent variable. The remaining two parameters are the nodal values for this spline. The first node of this spline is located at a consumed mass fraction of a value of .01 greater than the value of fuel consumed in the initial. maximum mass-flow rate burn. This slight off-set of the node value allows the mass flow rate (and hence the thrust) to be a continuous function of fuel-consumed fraction. A single-segment linear spline with the consumed fuel-mass fraction as the independent variable is used to ramp between the maximum thrust and the sustain-thrust burns. A typical profile is shown in Figure 3.2 . 


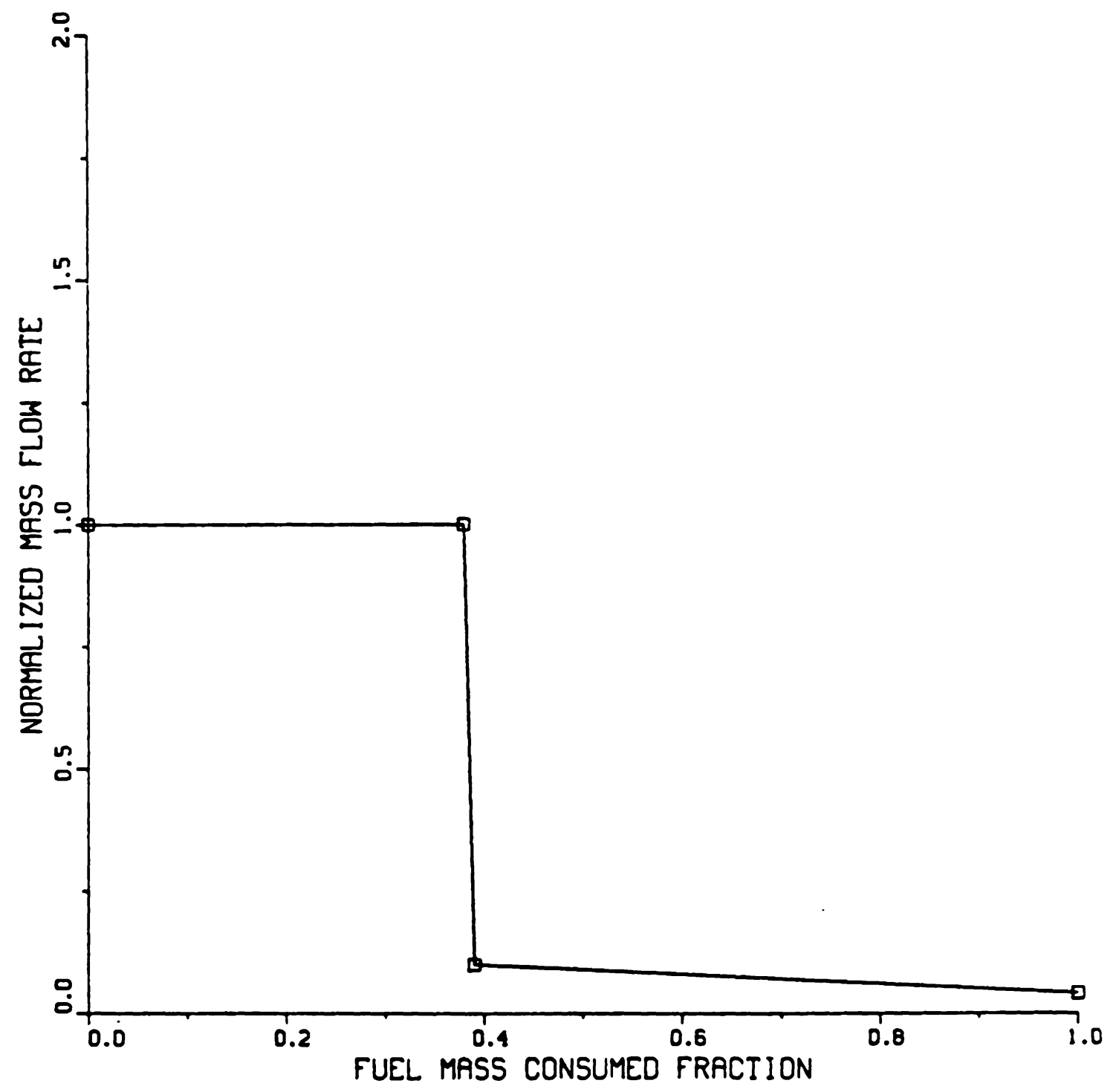

Figure 3.2 Typical Mass Flow Rate History 
The problem is to optimize the three motor parameters so as to maximize the total boost-glide range of the missile. Because of the extremely long glide times relative to the motor burn duration, the trajectory model terminates at motor burnout. In order to lower computational costs, the glide range is determined from a closed form expression. The total combined range of the boost and glide is then the quantity to be maximized.

\section{Trajectory Model}

The trajectory model for the continuously-variable-thrust horizontal-flight problem is relatively simple. Only two state variables, range and specific energy, are required. Since the trajectory is integrated from ignition to burnout, vehicle mass was selected as the independent variable. The system differential equations are given by the following expressions.

$$
\begin{aligned}
& \frac{d x}{d m}=\frac{V}{\beta} \\
& \frac{d E}{d m}=\frac{(T-D) V}{m g \beta}
\end{aligned}
$$

The thrust is a function of the only control $(\beta)$ as perfect expansion of the nozzle flow is assumed. Specifically, the thrust is given by:

$$
T=\beta V_{\text {。 }}
$$


The mass-flow rate is determined from a three-segment linear spline as a function of the vehicle mass. The shape of this spline is determined from the three motor parameters selected by the optimization algorithim. A typical mass-flow-rate control history may be seen in Figure 3.2. The aerodynamic drag is the only remaining force on the vehicle and is given by Equation 3.1 .

\subsection{Comparison of Analytical and Numerical Results}

The results from the analytical solutions to the problem of optimal thrust programming for a lifting rocket will be compared to the results of the parameter-optimization approximation. The initial conditions for the trajectory are given in Table 3.2. The problem is defined to occur at sea level which has an associated potential-energy value of zero. Final conditions are given in Table 3.3. The trajectories in the normalized mass and velocity plane from the parameter-optimization and the analytical solutions are shown in Figure 3.3. The burn-rate schedules with respect to consumed fuel fraction for both solutions are given in Figure 3.4. Both solutions include the maximum-burn-rate initial arc which the analytical solution continues until burnout. This is then followed by a glide to a final velocity of zero. At the intersection with the singular arc, the mass-flow rate of the optimal-control solution exhibits a discontinuity and is reduced from the maximum value to a very small value as given in Figure 3.4. The parameter-optimization solution terminates the maximum-rate burn at a fuel-consumed fraction of 0.36 which is reached before the intersection 


\section{TABLE 3.2}

Initial Conditions for the Continuously-Variable Thrust Horizontal Flight Problem

Quantity

Specific Energy

Downrange

Atmospheric Density

Vehicle Mass
Value

100.0 feet

0.0 feet

0.0023769 slugs $/ \mathrm{cu} \mathrm{ft}$

30.235 slugs 


\section{TABLE 3.3}

\section{Final Conditions for the Continuously-Variable Thrust Horizontal-Flight Problem}

Quantity

Specific Energy

Downrange

Vehicle Mass
Value

Free

Free

16.939 slugs 


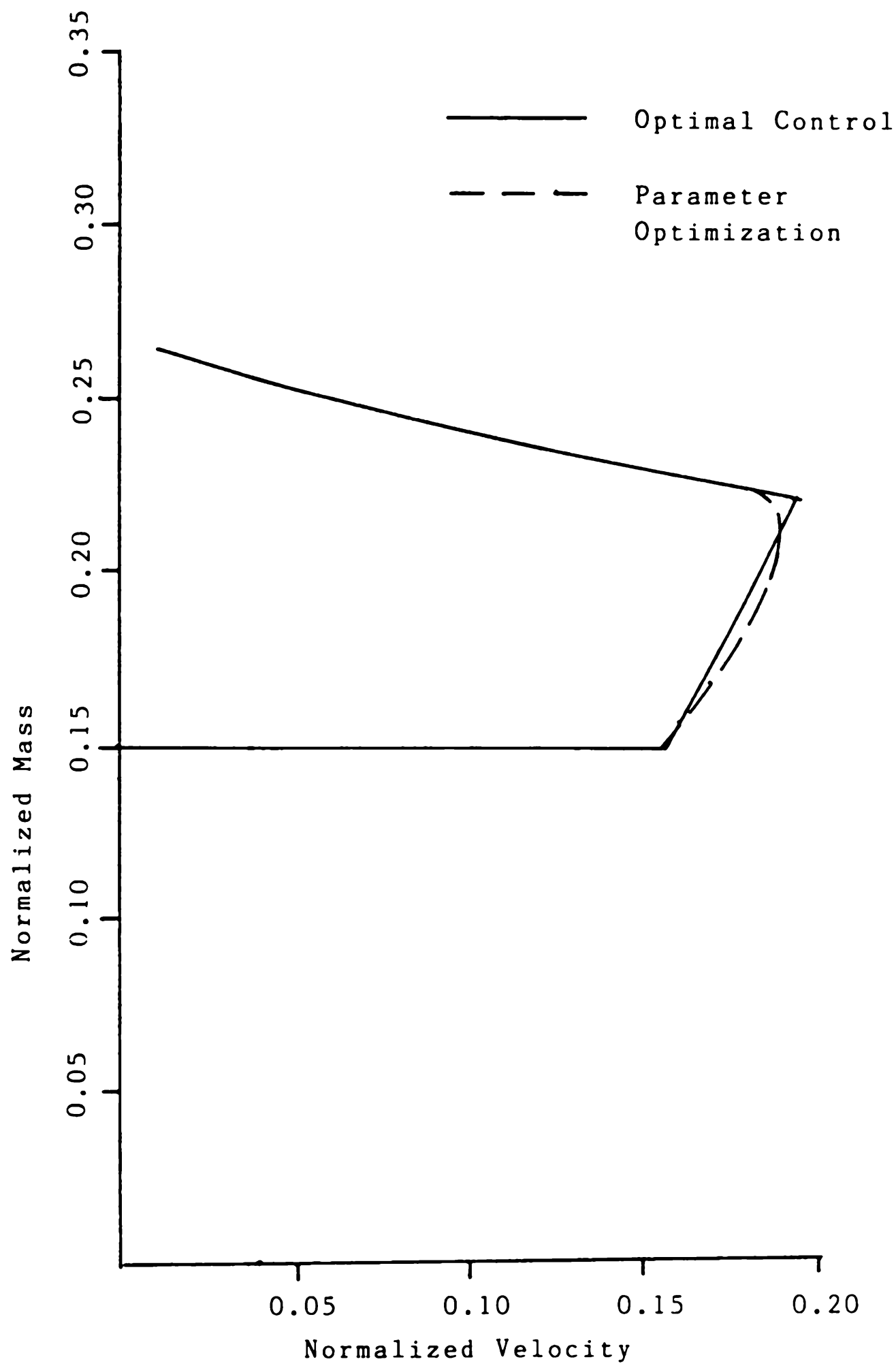

Figure 3.3 Optimal Control and Parameter Optimization Trajectories 


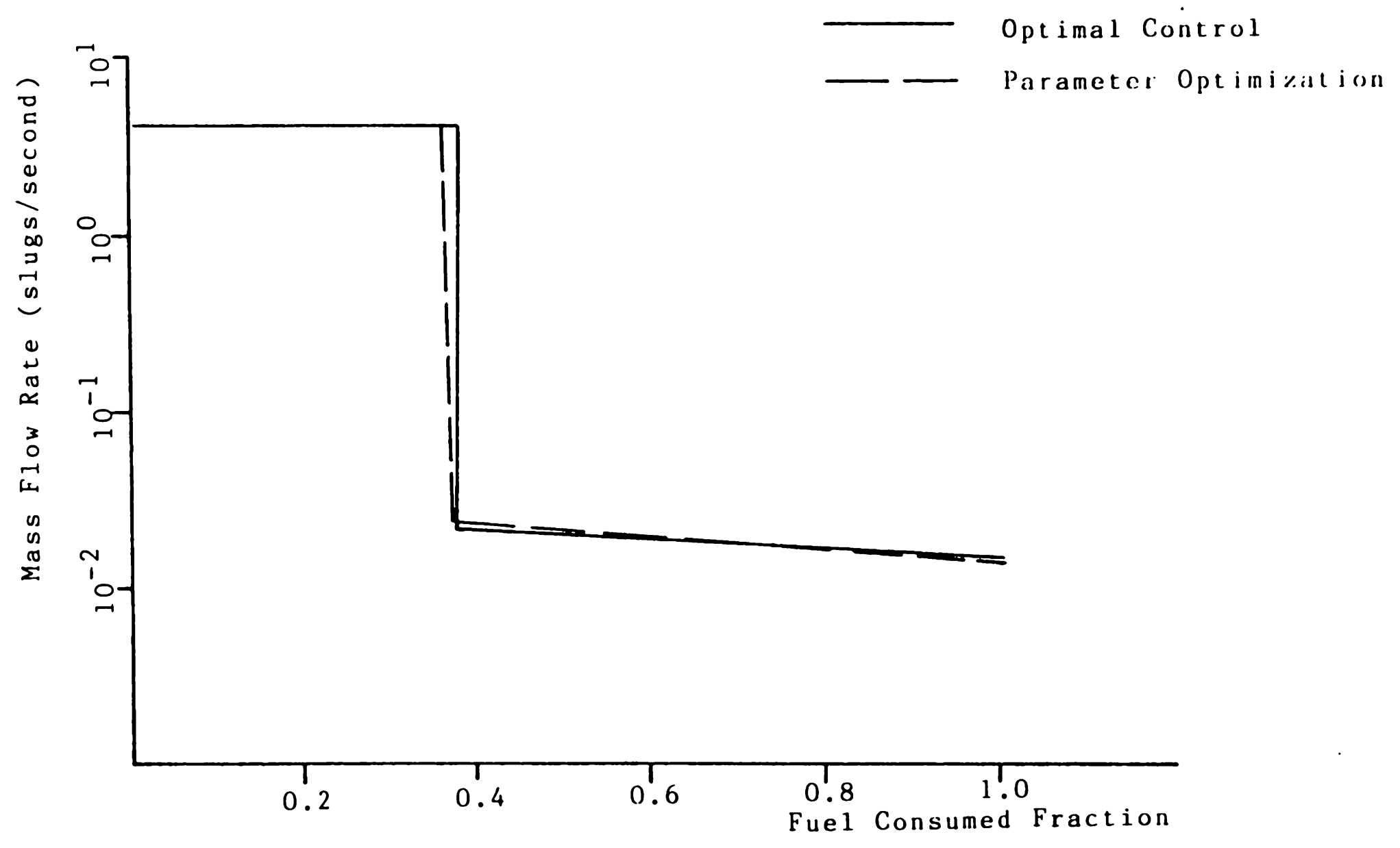

Figure 3.4 Optimal Control and Parameter Optimization Mass Flow Rate Histories for One Segment

Sustain Rate Spline 
with the singular arc. The parameter-optimization solution then follows a mass-flow rate schedule of slightly greater magnitude than the singular control. Thus, it passes across the singular arc into Region II of the normalized mass-velocity plane. In this region. burnout occurs and a coasting arc is followed until a zero velocity is achieved.

Ideally, the parameter optimization should yield a solution which closely approximates the analytical optimal-control solution. Approximations required for the parameterization may be the source of the discrepancies. Unlike the optimal-control solution, the parameterized model solution can not feature an instantaneous change in the burn rate from the maximum-rate burn. Instead, the burn rate must be ramped from the maximum value to the lesser-magnitude burn rate. This would have the effect of reducing the fuel-consumed fraction for termination of the maximum-rate burn. Furthermore, the sustain arc was represented by a single-segment linear spline which is a rather coarse approximation. To determine the effect of refining the sustain-arc grid, the parameter optimization was performed again with a two-segment linear spline for the sustain arc. As before, the first node of the spline was located at a fuel-mass-consumed fraction of .01 greater than the value in the initial-maximum thrust arc and the mass-flow rate was ramped over this spacing. The third node was placed at a fuel-consumed fraction of 1.0 or burnout and the second node was placed at a frac-tional value midway between these two points. This problem has four "degrees of freedom" for optimization instead of three and one would 
expect superior performance. The mass-flow rate schedule resulting from this new problem may be seen in Figure 3.5. There is an improvement in the fuel-fraction consumed in the maximum-thrust arc from 0.360 to 0.365, which more closely approximates the optimal-control solution. There is also variation in the nodal values of the sustain-arc spline. The first nodal value shows a slight increase relative to the one-segment sustain-arc solution while the second and third nodal values lower the value of the mass-flow rate on the sustain arc as compared to the one-segment sustain-arc solution. Thus, as the parameterization becomes of increasing fidelity the parameter optimization solution does approach the optimal-control solution. 


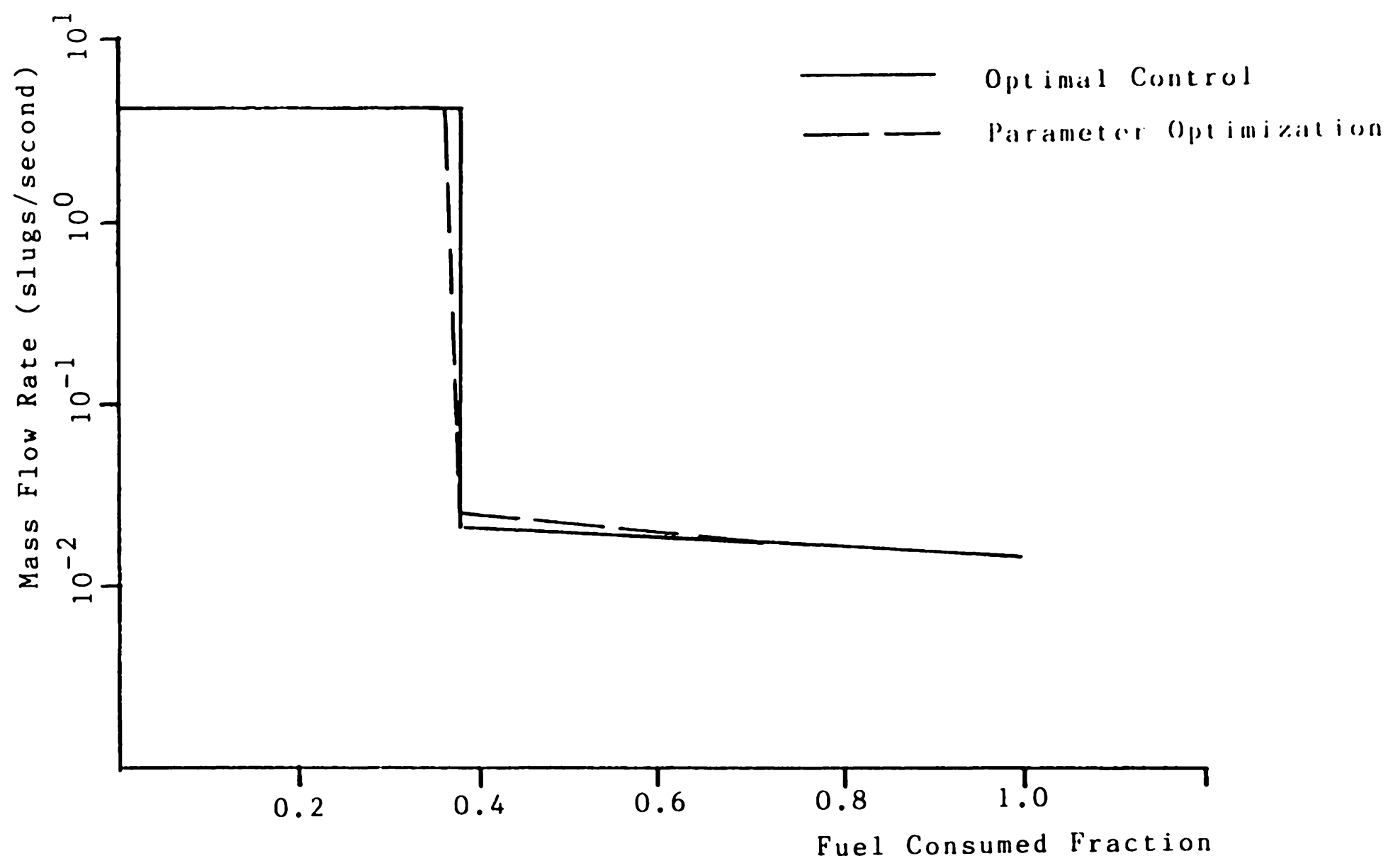

Figure 3.5 Optimal Control and Parameter Optimization Mass Flow Rate Histories for Two Segment

Sustain Phase Spline 


\section{Chapter 4}

Pulsed-Motor Trajectory Optimization

\subsection{Overview}

The problem considered in this chapter is that of optimizing the motor characteristics and the load-factor history for a single-stage, re-ignitable or pulsed solid rocket. Both horizontal- and elevation-plane trajectories will be considered. For the horizontal. one-dimensional problem. lift is equated to weight, leaving only the motor characterizations available for optimization. Both two- and three-pulse motors will be included in the study with the motor characteristics selected so as to maximize the speed of the missile at a specified final range. Final time is free. The elevation-plane problem will be to select the load-factor history and motor parameters of a oneand a two-pulse, solid-fuel, single-stage surface-to-air missile to maximize the velocity at a fixed final altitude and range. The effect of various maximum time-of-flight constraints is also studied. A second elevation-plane problem is studied. This problem consists of selecting the load-factor history and motor parameters which minimize the time-of-flight to a specified final altitude and range. This last problem is important since it yields a lower bound on time-of-flight, which is constrained in the former problem. 


\subsection{Parameterization}

This section will discuss the characteristics of both the two- and three-pulse motor configurations and the selection of the motor parameters for optimization. The motor is a solid-fuel, fixed mass-flow-rate configuration with a fixed total-fuel mass. Thus, the mass-flow rate is constant over each of the rectangular thrust pulses. The shape of the mass-flow-rate history for a specific set of parameter values may be seen in Figure 4.1. For the three-pulse motor, the mass-flow-rate history is defined by four parameters which determine the fraction of the fuel allocated to each pulse and the ignition time of each pulse. Two parameters are required to describe the fuel allocation between the pulses. The first parameter is defined to be the fuel fraction of the first pulse. The second parameter is defined to be the fraction of fuel remaining after burnout of the first pulse, which is allocated to the second pulse. The fuel allocation of the third and final pulse is simply the remaining fuel after the burnout of the second pulse. The remaining two parameters determine the ignition times of the last two pulses. The ignition time of the first pulse is fixed at launch. The burn time of each pulse is fixed by the fuel allocation of the pulse and the fixed burn-rate. Thus, the interpulse coast times are the only temporal factors available for optimization. The first temporal parameter is defined to be the fraction of selected total time-of-flight which is to occur during the coast between the burnout of the first pulse and ignition of the second pulse. The second temporal parameter is defined to be the fraction of total remaining coast time 


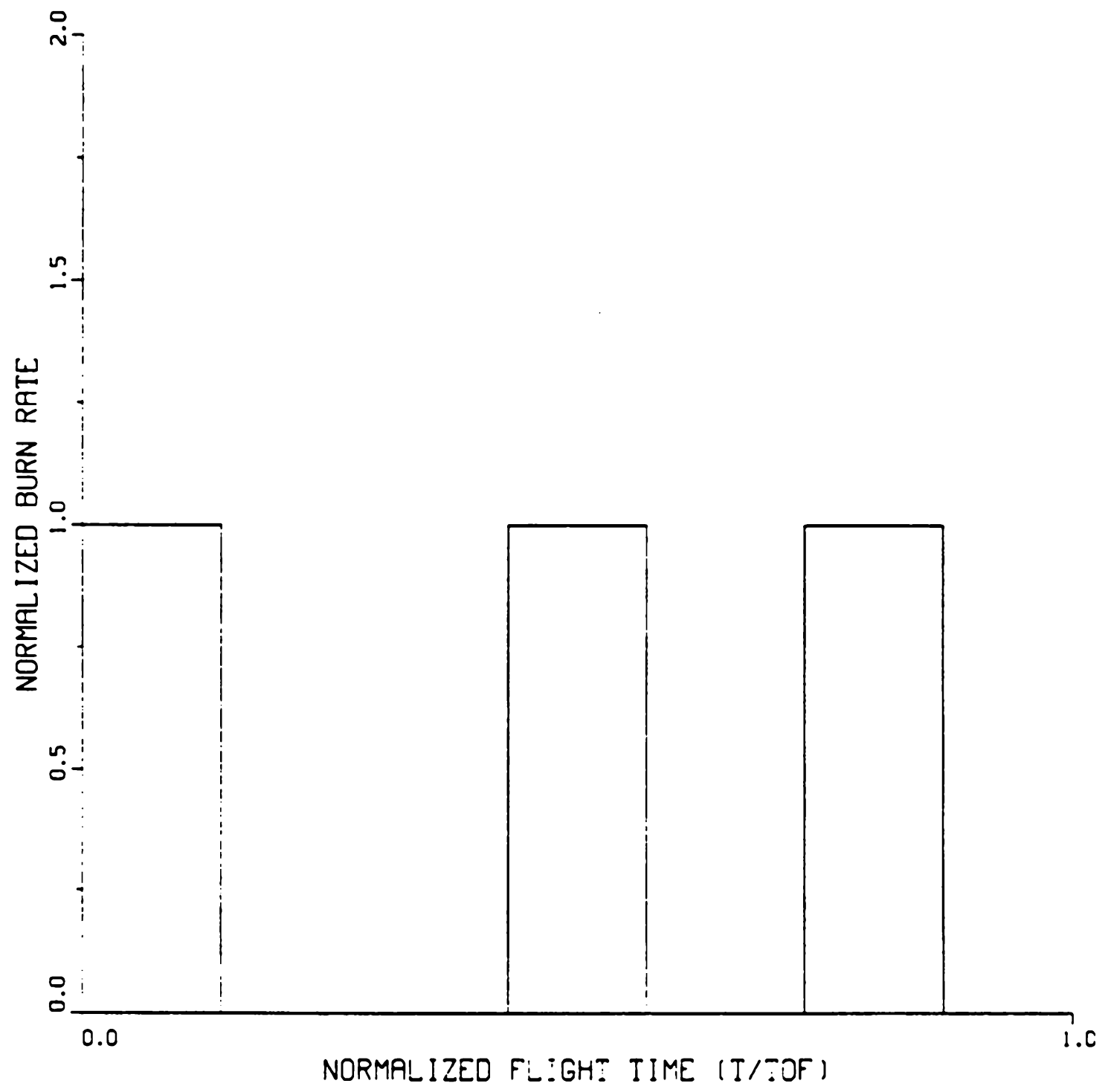

Figure 4.1 Burn Rate History for Three Pulse Motor 
after burnout of the second pulse to be used in the coast between the burnout of the second pulse and the ignition of the third. The total remaining coast time is determined by subtracting the burn times of each of the three pulses and the interpulse coast time between the first and second pulses from the selected total time-of-flight. Thus, these two parameters are sufficient to fix the ignition and burnout times of each pulse. Any remaining coast time represents coasting flight after burnout of the third pulse until the total time-of-flight is achieved. The three-pulse motor has a total of four parameters which may be selected to optimize the performance index, while the two-pulse motor has only two. These two parameters for the two-pulse motor configuration include the fuel fraction of the first pulse and the time-of-flight normalized interpulse coast time. The fuel fraction of the second pulse then consists of all remaining fuel after burnout of the first pulse.

After the propulsion parameters, the next parameter to consider is the time-of-flight. The optimization algorithim determines the time-of-flight as one of the parameters optimized. The importance of the time-of-flight parameter is that it determines the termination of the trajectory. The altitude and range which the missile has achieved at that point are defined as the final values for the optimization algorithim. This is also true of the value of the performance index achieved at the termination of the trajectory model. Thus, to ensure that the specified altitude and range are achieved, equality constraints are placed upon the final altitude and range. 
While the horizontal-flight problem has only the thrust-associated parameters and the total time-of-flight available for optimization. the elevation-plane trajectory problem has an additional set of parameters associated with the load-factor control history. The load-factor history is approximated by a multi-segment linear spline with normalized time-of-flight as the independent variable. The value of the load factor at each of the node points of this spline constitute the remaining parameters available for optimization of the elevation-plane trajectories. The temporal positions of these node points are defined by a user-selected set of time-of-flight normalized values. Thus, as the time-of-flight parameter increases or decreases, the node schedule expands or contracts accordingly. This allows each trajectory to have the same number of load-factor spline nodes and prevents any of the parameters associated with the load-factor history from becoming irrelevant to the optimization problem. These normalized temporal node positions begin with a value of 0.0 and terminate with a value of 1.0. The spacing and number of node points is selected by the user. A set of five nodes is used in the present study as seen in Figure 4.2 for a specific set of node values.

The trajectory model is designed so that there is no difference between the commanded load factor and the achieved load factor. For the elevation-plane trajectory problem the optimization algorithim may select load factors and thrust parameters which produce velocity profiles requiring lift coefficients which exceed the maximum achievable value for the airframe. The possibility also exists for the horizontal 


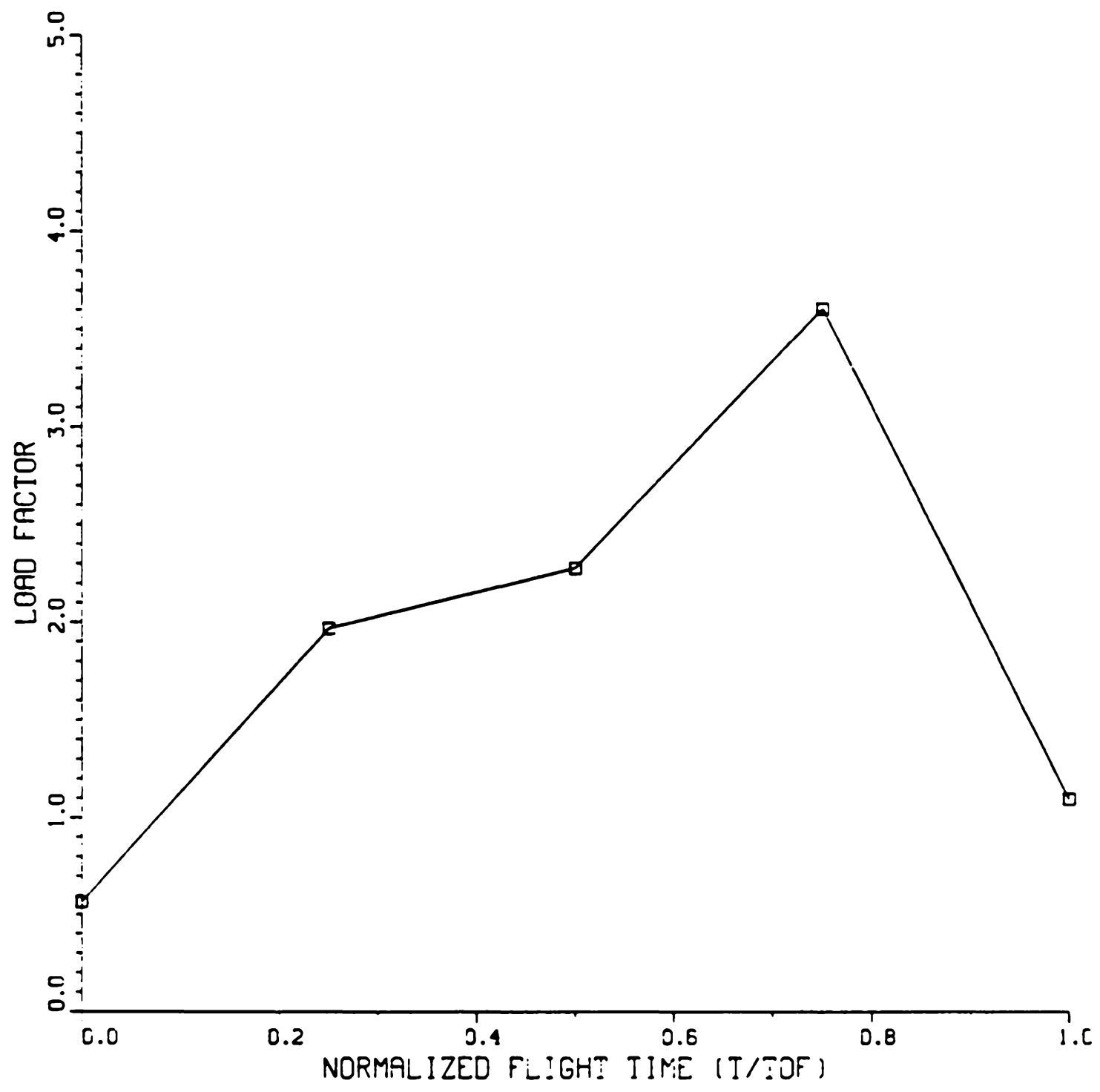

Figure 4.2 Typical Load Factor History 
problem in that velocity profiles may result which require lift coefficients which exceed the maximum achievable value in order to meet the level-flight requirement. To prevent the violation of the maximum lift coefficient constraint, an additional state variable, $E_{2}$, is used to measure the violation of the maximum achievable lift coefficient in terms of an integral penalty (7). The derivative of this variable is given by the following relation.

$$
\begin{aligned}
& \dot{E}_{2}=0 \text { if } C_{L} \leq 0.95 C_{L \max } \\
& \dot{E}_{2}=\left(C_{L}-0.95 C_{L \text { max }}\right)^{2} \text { if } C_{L} \geq 0.95 C_{L_{\max }}
\end{aligned}
$$

\subsection{Trajectory Mode 1}

The results of a trajectory simulation and thus those of the optimization algorithim using this simulation depend upon the mathematical models used to describe the dynamics of the vehicle. This section presents the details of the simulation and the assumptions used in its development. The trajectory simulation is an elevation-plane, point-mass model with trim aerodynamics. Rotational dynamics are assumed fast enough to be negligible. The dynamics of the missile are characterized by the specific energy, $E$, the altitude, $h$, the range-coordinate, $x_{0}$ the missile mass, $m$, and the flight path angle, $\gamma$. The thrust is assumed to act along the velocity direction. The resulting equations of motion are given by the following system. 


$$
\begin{aligned}
\dot{E} & =\frac{(T-D) V}{m g} \\
\dot{h} & =V \sin \gamma \\
\dot{x} & =V \cos \gamma \\
\dot{m} & =-\beta \\
\dot{\gamma} & =(L / m g-\cos \gamma) g / V
\end{aligned}
$$

The forces on the missile include the lift, drag, and thrust, the details of their computation to follow. The lift is a direct function of the commanded load-factor obtained by means of a multi-segment linear spline through the selected parameter values at each node point. The aerodynamic drag is a function of the Mach number and the lift coefficient and is determined from a cubic-spline lattice (8) fit through the aerodynamic data presented in Appendix A. Additional data are included deliberately in the spline fit to extend the range of data beyond the limits to be encountered in the use of the optimization algorithim. Atmospheric density and local sonic velocity are determined by the use of cubic splines with altitude as the independent variable. The thrust computation includes both the momentum and pressure thrust effects. The thrust is then given by the following relation.

$$
T=\beta V_{e}+\left(P_{e}-P_{a}\right) A_{e}
$$


The mass-flow rate at any time is determined by the selected motor parameters. If the current time is between the ignition and burnout times of a pulse then the mass-flow rate is set equal to the value in Appendix A. Otherwise, the mass flow rate is nulled and the exhaust pressure is equated to the ambient pressure.

The integration step-size in the trajectory model is variable and is selected so as to ensure smoothness in the value and the first derivative of the performance index and the constraint functions. Each trajectory contains one hundred integration steps with the nominal step-size determined by the partitioning of the total time-of-flight into one hundred equal-sized steps. Thus, the value of the nominal step-size varies with the total time-of-flight. To ensure smoothness of the performance index and the constraint functions, it is necessary that the appropriate integration steps coincide with discontinuities in the missile dynamics. These discontinuities arise from two sources: the engine event times; and, the node points in the load-factor history. To ensure smoothness at the load-factor node points, the normalized temporal coordinates of the load-factor spline nodes are defined such that they coincide with integral multiples of the normalized nominal time-step of .01. To avoid discontinuities in the thrust profile occurring during an integration step and since engine event times may vary for each trajectory, logic must be implemented to prevent an engine event is scheduled to occur within the next integration step. the step is shortened to coincide with the upcoming event. The next step is then 
sized to place the following time step on the next integral multiple of the nominal step.

\section{4 Horizontal Pulsed Flight}

The horizontal flight problem involves the maximization of the final velocity of a missile with a non-zero initial velocity. The missile must fly with lift equal to weight to a specified range location at the same altitude. The one-, two-, and three-pulse motor configurations are all considered and each has the same initial conditions and final range requirement (see Table 4.1). Since lift is specified by the level-flight requirement, only the motor parameters may be selected. For the one-pulse motor there is one parameter, total time-of-flight, and one equality constraint, the final range, leaving no freedom available for optimization. For the two-pulse motor configuration there are two motor parameters, the total time-of-flight parameter, and the final range equality constraint, giving a net of two "degrees-of-freedom" for the optimization. The three-pulse motor configuration has four motor parameters, the total time-of-flight parameter, and the single equality constraint on final range which allows the optimization procedure four "degrees-of-freedom". In addition to the equality constraints, the horizontal-flight problem also has a number of inequality constraints. For the one-pulse motor configuration, the only inequality constraint is the integral penalty on the lift coefficient history as seen in Table 4.2. The two-pulse motor has in addition to the integral penalty on lift coefficient a number of 
TABLE 4.1

Initial and Final Constraints for Horizontal Pulsed Flight

Quantity

Initial Specific Energy

Initial Altitude

Initial Downrange

Final Specific Energy

Final Altitude

Final Downrange

Final Flight Path Angle
Value

25000.0 feet 20000.0 feet

0.0

Plaximal

20000.0 feet

150000.0 feet

0.0 


\section{TABLE 4.2}

Two-Pulse Horizontal-Flight Inequality Constraints

Quantity

Integral Lift

Coefficient Penalty

Fuel Fraction

First Pulse

Normalized Burnout

Time of Second Pulse

Normalized Interpulse

Coast Time
Constraint

$\leq 0.5$

$\leq 0.8$

$\geq 0.1$

$\leq 1.0$

$\geq 0.1$ 
constraints on the motor parameters as given in Table 4.2. The three-pulse motor configuration has a number of inequality constraints including the lift-coefficient integral penalty and the motor constraints given in Table 4.3. These constraints, while not necessarily active in the solution, are selected to prevent the problem from becoming ill-conditioned during the search process.

The optimal parameters for both the two- and three-pulse motor configurations are given in Table 4.4. Although no optimization was required, the associated characteristics of the one-pulse motor configuration are also included in the table. The terminal results for each of the pulse configurations are presented in Table 4.5. Velocity histories for each configuration are given in Figure 4.3 through 4.5. By avoiding the high post-boost velocities associated with the single-pulse motor configuration, the use of the two-pulse motor produces almost a 163.0 percent increase in terminal velocity over the one-pulse motor configuration. The use of three pulses produces a 206.6 percent increase over the baseline single-pulse motor. The peak velocities for both the two-pulse and three-pulse motor configurations occur just prior to the termination of the flight where the energy loss due to drag will be minimal. For the one-pulse motor, the peak speed of 4220.0 feet per second occurs at the end of the boost early in the flight where the high dynamic pressure and drag drain off energy. For the three-pulse motor configuration, the speeds after the first and 
TABLE 4.3

Three-Pulse Horizontal-Flight Inequality Constraints

Quantity

Integral Lift Coefficient Penalty

Fuel Fraction

First Pulse

Fraction of Remaining Fuel in

Second Pulse

Normalized Burnout Time of Third Pulse

Normalized First Interpulse Coast Time

Normalized Second Interpulse Coast Time
Constraint

$\leq 0.5$

$\leq 0.8$

$\geq 0.1$

$\leq 0.8$

$\geq 0.1$

$\leq 1.0$

$\leq 1.0$

$\geq 0.1$ 
TABLE 4.4

Parameter Values for Optimal Horizontal-Pulsed Flight

Motor

Single

Double

Triple
Fuel Fractions Pulse No.

12

3

$1.0-\quad-$

$.532 .468 \quad-$

$\begin{array}{lll}.265 & .184 & .551\end{array}$
Pulse Ignition Times Pulse No.

2

3

- $\quad-$

115.4

67.5

132.2
TOF

72.5

121.8

135.6 


\section{TABLE 4.5}

Final Values for the Optimal Horizontal-Pulsed Flight

$\begin{array}{lccc}\text { Motor } & \begin{array}{c}\text { Final Energy } \\ (\text { feet })\end{array} & \begin{array}{c}\text { Final Velocity } \\ (\text { feet/sec })\end{array} & \begin{array}{c}\text { Final Speed } \\ (\text { feet/sec })\end{array} \\ \text { Single } & 36,300 . & 1025 . & 2068 . \\ \text { Double } & 133,000 . & 2697 . & 1231 . \\ \text { Triple } & 173,000 . & 3143 . & 1074 .\end{array}$


52

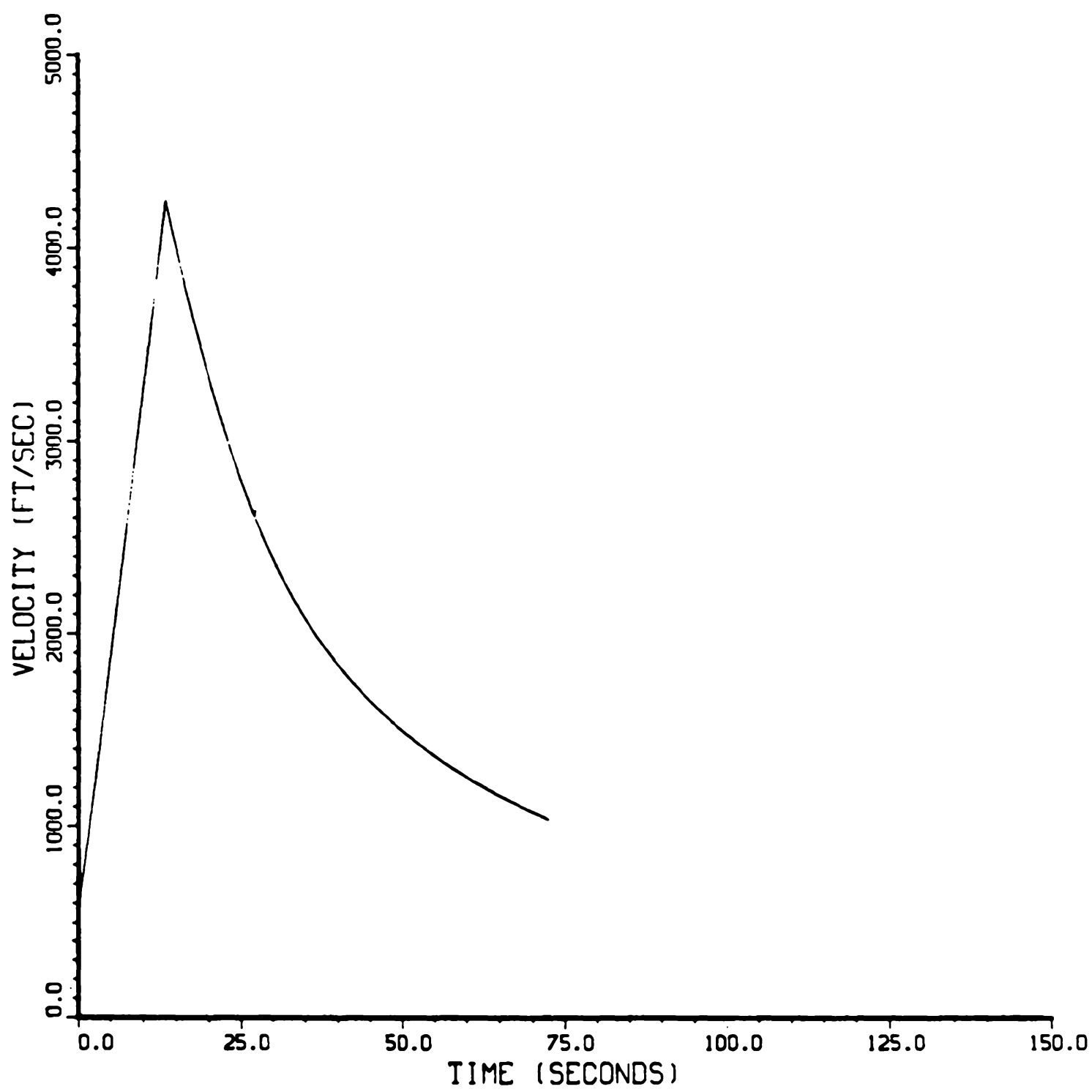

Figure 4.3 One-Pulse Horizontal Flight Velocity History 


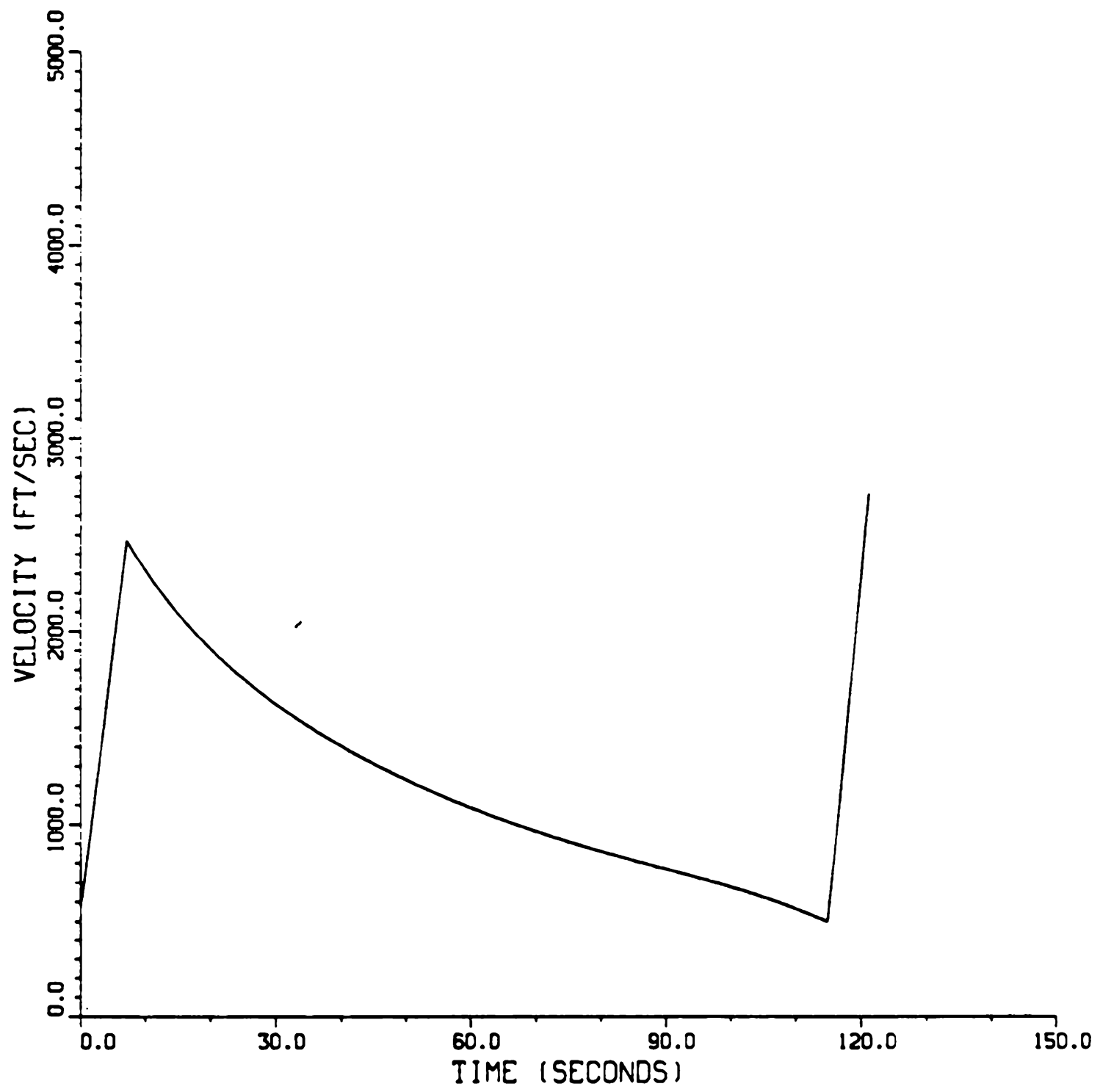

Figure 4.4 Two-Pulse Horizontal Flight Velocity History 


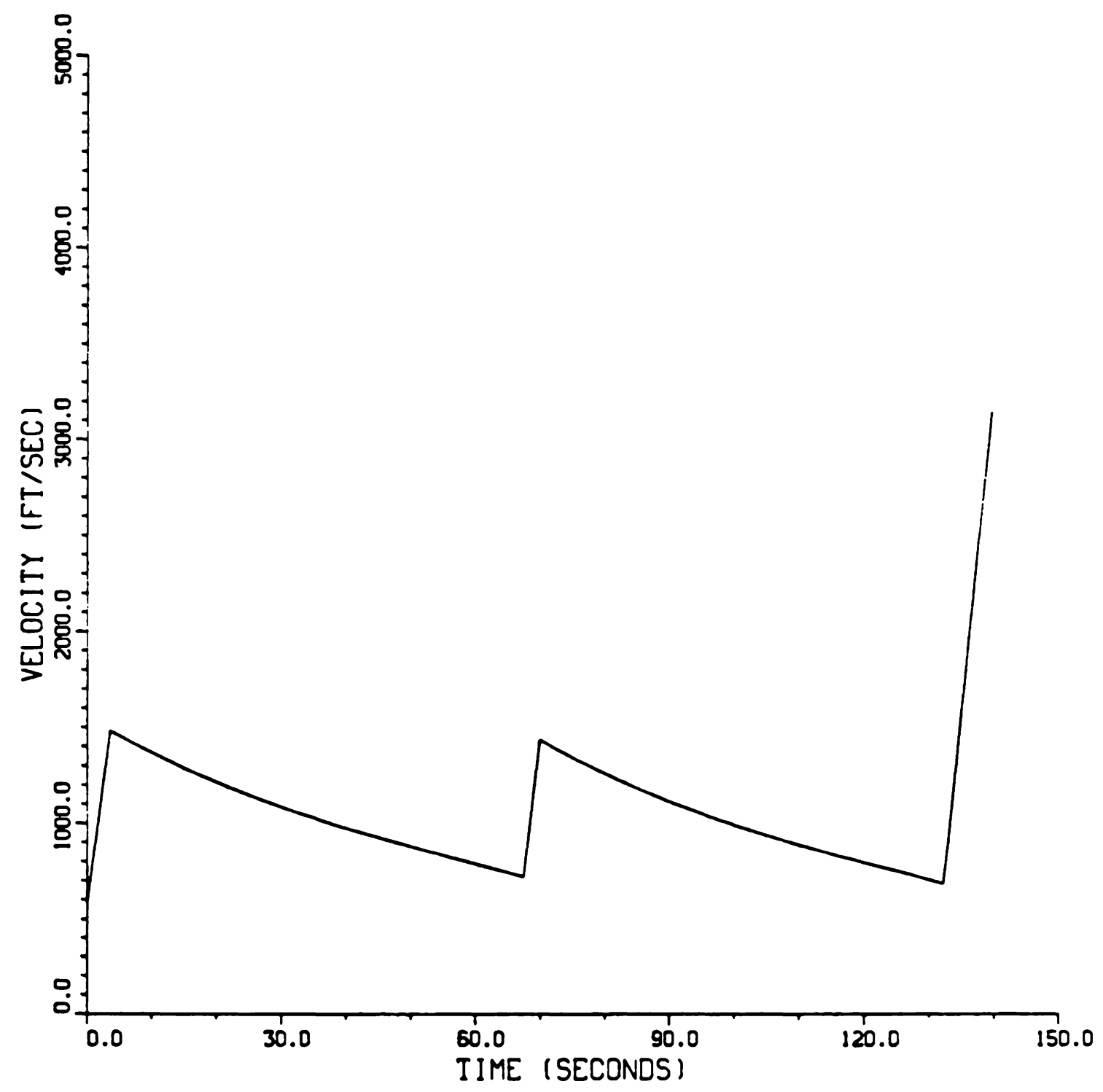

Figure 4.5 Three-Pulse Horizontal Flight Velocity History 
second pulse are less than the speed after the first pulse of the two-pulse motor. This allows the three-pulse motor configuration to reach the final range with less fuel consumed to overcome aerodynamic drag and thus more remains to be burned in the terminal pulse. The three-pulse configuration has an almost even division of the trajectory in terms of time between the two non-terminal pulses. One would expect this pattern to continue as more pulses are added with the speed after each pre-terminal pulse decreasing and the re-ignition speed increasing. Because of the binary nature of the thrust control, one would expect that as the number of pulses approached infinity the result would be a form of chattering control about some singular velocity history associated with the optimal control solution (2). This solution consists of an initial maximum thrust burn coupled to a singular control arc with an intermediate mass-flow rate followed by a final coasting phase. Thus, as the number of pulses is increased it becomes possible for the pulse-motor configuration to more closely approximate the intermediate-throttle operation associated with the singular control. For both the two- and three-pulse motors the pre-terminal peak speeds of 2475.0 and 1471.0 feet per second, respectively, occur at the end of the first pulse. The multi-pulse motors offer pre-terminal peak speed reductions of 41.0 and 65.0 percent. This ability to reduce peak speed not only gives the advantage of increasing the terminal velocity and energy but also allows the avoidance of aerothermal problems associated with high Mach numbers for certain missile materials. All of these benefits come at the costs of decreased mean ground speed. The mean ground speed of the three-pulse motor represents a 40.0 percent 
reduction of the mean ground speed as compared to the one-pulse motor configuration.

Another interesting feature of the multi-pulse motor configurations is the development of the pulsing structure as pulses are added. The two-pulse motor has an almost even allocation of fuel between the pulses. After the first pulse, the missile coasts until there is just enough time to burn all of the remaining fuel. The three-pulse motor configuration also has approximately half of the fuel in the terminal pulse while the remainder is split between the first and second pulses. The velocities at ignition of the second and third pulse are nearly equal at 724.0 and 684.0 feet per second, respectively, and are greater than the re-ignition speed problem with the given initial and final conditions and with the final time free, the optimal trajectory would be expected to involve all of the pulses allowed.

With the given initial and final conditions, the optimal parameters all have midrange values and thus do not approach the constraints. As these end-conditions vary, the optimal pulsing structure will vary and may encounter the constraints. As the initial speed of the missile is decreased, the fuel allocated to the first pulse will increase and may eventually violate the upper bound on the fuel fraction of the first pulse. Conversely, increasing the initial speed will cause the fuel allocation of the first pulse to decrease until it approaches the lower bound. 


\subsection{Elevation-Plane trajectories}

The elevation-plane trajectory problem involves maximizing the velocity of a vertically-launched, single-stage, solid-fuel surface-to-air missile at a fixed final altitude and range. Since the final altitude is specified, maximizing the final velocity is equivalent to maximizing the final energy of the missile. Both one- and two-pulse motor configurations are analyzed. For the one-pulse motor, only the final-time-free problem is studied, while for the two-pulse motor configuration the effect of a final-time constraint is also considered. No other final time-constraints are placed on the solution. In addition to the problem of maximizing the final velocity at a specified terminal point, the problem of minimizing the total time-of-flight to a specified final altitude and range is presented for both the one- and two-pulse motor configurations. The initial conditions are both the one- and two-pulse motor configurations are given in Table 4.6. The final conditions for both configurations are given in Table 4.7.

The single-pulse motor configuration has all of its motor characteristics fixed, leaving only the time-of-flight and the load-factor spline node values to be optimized. Thus, the single-pulse configuration has a total of six parameters available along with the t:yo equality constraints on final altitude and range netting four "degrees of freedom" for optimization. For realistic vehicle operation, the resulting load-factor spline node values must not result in the violation of the maximum lift coefficient value which is enforced by an integral square penalty term. For the one-pulse motor, this is the only 
58

TABLE 4.6

Initial Conditions for Elevation-Plane Trajectories

Quantity

Altitude

Energy

Range

Flight Path Angle
Value

0.0 feet

200.0 feet

0.0 feet

1.571 radians 
59

TABLE 4.7

Final Conditions for Elevation-Plane Trajectories

Quantity

Altitude

Energy

Range

Flight Path Angle

Time
Value

70000.0 feet

Maxima 1

120000.0 feet

Free

Free/Constrained 
control constraint. The two-pulse motor configuration problem has not only the five load-factor-spline-associated parameters and the time-of-flight parameter, but also the two motor parameters available for optimization. These two motor parameters are the same as for the two-pulse, horizontal-flight problem and consist of the fuel fraction in the first pulse and the normalized interpulse coast time. In addition to the two equality constraints on final altitude and range, the two-pulse motor problem has five motor-parameter inequality constraints and the integral-square-penalty constraint on the lift coefficient as seen in Table 4.8. Thus, the two-pulse motor configuration elevation-plane trajectory problem has what may be considered six "degrees of freedom" available for optimization. Of course, as any of the inequality constraints become active this will reduce the freedom of the optimization procedure.

Some results of the optimization of the one- and two-pulse motor configurations with the final time free are presented in Table 4.9. The terminal conditions for both of these trajectories may be seen in Table 4.10. The shape of the two trajectories may be seen in Figure 4.6 while Figure 4.7 has the velocity-range history.

The gain in performance for the two-pulse motor relative to the one-pulse motor is not as dramatic as it was for the horizontai-flight problem. The terminal energy showed only a 13.0 percent increase shile the terminal dynamic pressure increased by 36.6 percent. The optimal two-pulse motor configuration for the specified final altitude and range has a little more than one-half of the total fuel in the first pulse. 
TABLE 4.8

Nominal Two-Pulse Elevation-Plane Problem Constraints

Quantity

Integral Lift Coefficient Penalty

Fuel Fraction

First Pulse

Normalized Burnout Time of Second Pulse

Normalized Interpulse Coast Time
Constraint

$\leq 0.5$

$<0.9$

$>0.1$

$\leq 1.0$

$>0.0$ 


\section{TABLE 4.9}

Elevation-Plane Optimization Parameter Values

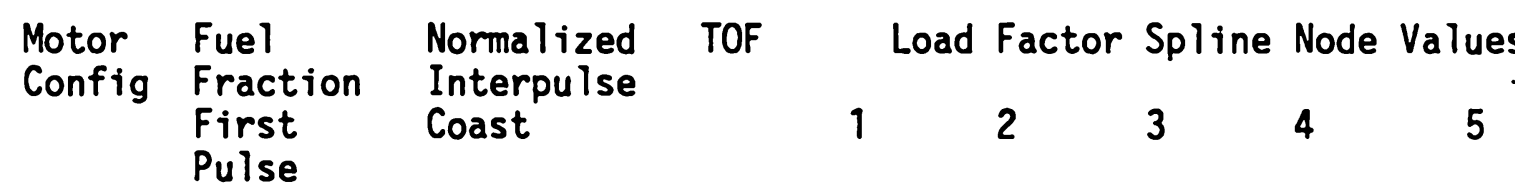

$\begin{array}{lllllllll}\text { Single } & 1.0 & - & 68.84 & -.349 & -3.41 & -.927 & -.446 & -.138 \\ \text { Double } & .550 & .377 & 75.12 & -.810 & -.683 & -.136 & -.647 & .0432\end{array}$


TABLE 4.10

Optimal Single and Double Pulse Elevation Plane Trajectory Final Values

$\begin{array}{ccccc}\begin{array}{c}\text { Motor } \\ \text { Configuration }\end{array} & \begin{array}{c}\text { Energy } \\ \text { (feet) }\end{array} & \begin{array}{c}\text { Final Velocity } \\ \text { (feet/second) }\end{array} & \begin{array}{c}\text { Mean Ground Speed } \\ \text { (feet/second) }\end{array} & \begin{array}{c}\text { Flight Path } \\ \text { Angle } \\ \text { (Radians) }\end{array} \\ \text { Single Pulse } & 141.000 . & 2140 . & 1861.0 & -.2327 \\ \text { Double Pulse } 167,000 . & 2500 . & 1597.0 & -.0407\end{array}$




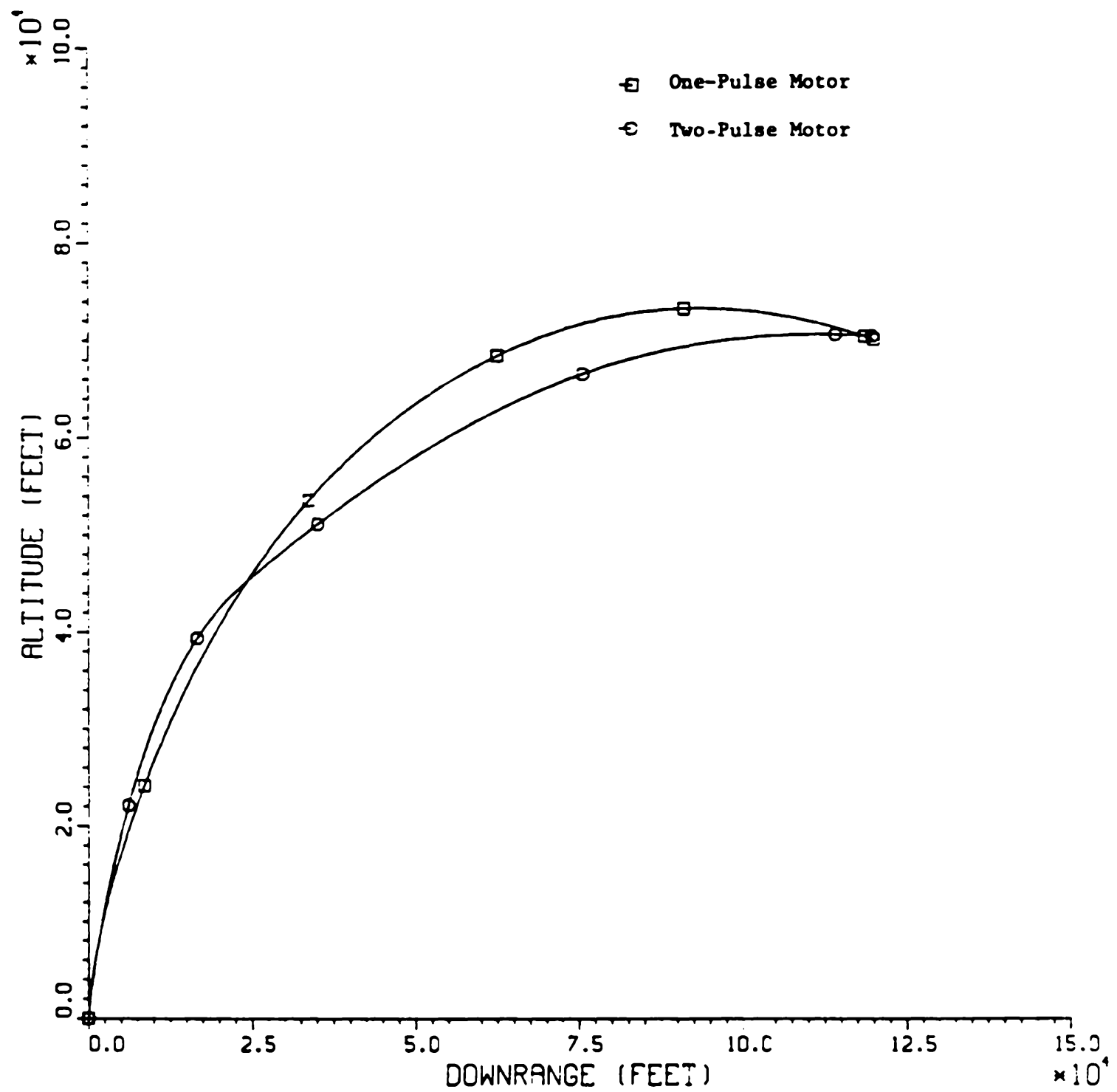

Figure 4.6 Elevation Plane One- and Two-Pulse Trajectory Shapes 


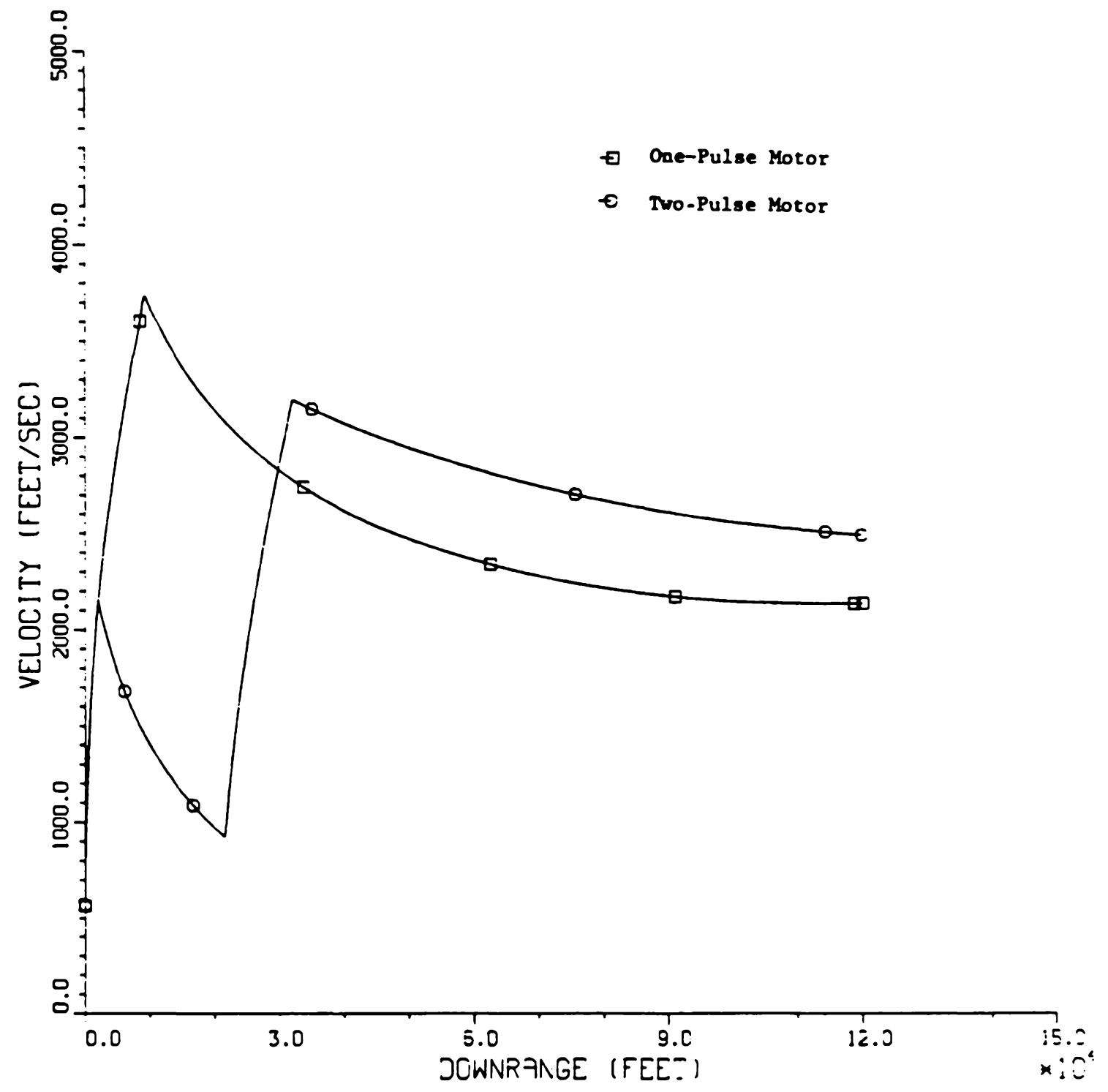

Figure 4.7 Elevation Plane One- and Two Pulse Velocity Histories 
By delaying ignition of the second pulse until 35.73 seconds, the two-pulse motor configuration exhibits better energy management than the one-pulse case by coasting until the dynamic pressure decreases through the value where the lift-to-drag ratio peaks. Reignition occurs at a dynamic pressure of 436.0 pounds per square foot and a Mach number of .956. It should be noted that the second pulse does not occur at the end of the flight as it does for the two-pulse horizontal-flignt problem, but instead near the mid point. This is because of the gravity penalty associated with lifting the unburned fuel to higher altitudes. The gravity penalty must be traded off with the drag penalty associated with high-dynamic-pressure flight to produce the optimal pulsing structure. The increase in terminal energy for the two-pulse motor is directly related to the reduction in peak dynamic pressure. This increase in terminal energy and dynamic pressure does come at a cost. The reduction of speeds early in the trajectory for the two-pulse motor causes a 13.7 percent reduction in the mean ground speed to the terminal point. Secause of the high boost speed for the one-pulse motor the maximum load factor occurs during the high-speed regime and is significantly greater than the maximum value for the two-pulse motor, which occurs at launch. This very large increase in the magnitude of the commanded load factor for the one-pulse motor compared to the two-pulse motor has two major effects which include an increase in drag with a commensurate decrease in final energy and creating a trajectory with greater curvature. This is the reason that the one-pulse motor configuration has the steeper final flight-path angle. 
For both motor configurations, none of the inequality constraints were active. As might be expected the lift-coefficient history along the optimal trajectories has a magnitude much less than the maximum value. The motor parameters for the two-pulse motor had optimal values in the midrange and thus did not encounter either the upper or lower bounds. Of course, the optimal values of the parameters will vary as different initial conditions are defined. In that case, some of the control constraints may become active.

Often in surface-to-air missile applications, the fire control logic directs the missile to a target point in the sky. At this point. the missile would activate its terminal homing system to acquire and seek the target aircraft. Increased dynamic pressure of the missile at the target equates directly to greater maneuverability. The greater the interceptor maneuverability the greater the probability of a successful intercept. But often limits on the fire-control system place upper limits on the time available to fly to the target point. If the effect of pulsing the motor on the mean ground speed of the missile is considered to be detrimental to the overall mission performance, one might investigate the optimum pulsed solution under a varying maximum-time-of-flight constraint. Thus, trading final velocity for reduced flight time is an important issue. The time of flight considerations are evaluated by means of studying two additional problems. The first problem addressed is one of determining the effect on the final velocity value of including the requirement that the missile reach the specified final altitude and range in the minimum time 
possible. The approach used is to redefine the performance index to be the negative of the time-of-flight to the specified range and altitude. This minimum-time-of-flight problem is studied for both one- and two-pulse motor configurations. For the one-pulse motor configuration the constraints are the two equality constraints on the final altitude and the one inequality constraint on the lift history as given in Table 4.8. With the five load-factor nodal value parameters this allows three optimization "degrees of freedom" provided that the inequality constraint remains inactive. For the two-pulse motor configuration, the constraints on the problem are the final altitude and range requirements along with the motor and lift-history constraints given in Table 4.8. The parameters available for optimization are the same as defined earlier for the two-pulse motor configuration elevation-plane trajectory problem. These include the two motor-associated parameters, the total time-of-flight, and the five load-factor nodal value parameters. $1 ! i$ th the two equality constraints on final altitude and downrange, there are basically five optimization "degrees of freedom" for this problem provided that the inequality constraints remain inactive. It is interesting to note that the minimum-time trajectory involves a very short interpulse coast of 4.52 seconds. In spite of the coast, the two-pulse trajectory is faster than the fastest one-pulse trajectory which is of a 62.25 second duration. The two-pulse motor configuration produced the shortest time-of-flight and this may be considered as a lower bound on the time-of-flight out to the target point. 
The next problem addressed is that of determining the load factor and motor parameters which maximize the final velocity as with the free-final-time problem but with the final time-of-flight constrained to some maximum value. Thus, the minimum-time-of-flight trajectory serves as a lower bound. Any time-of-flight constraints less than the minimum value will fail to produce a solution. This problem is addressed by the addition of an inequality constraint on the maximum allowable time-of-flight to the final-time-free problem already presented. !: hile some mean speed freedom is available for the one-pulse motor configuration, it is not significant, so that only the tiwo-pulse motor configuration will be considered. It would be expected that as the mean ground speed increased from the final-time-free value that the energy and the velocity at the final time would decrease. Thus, one is faced with the prospect of trading off final velocity for mean ground speed. To verify this assumption, the time-constrained, two-pulse motor configuration problem was run with five time constraints less than the optimum time-of-flight. Intuition would lead one to believe that the maximum time-of-flight constraint would be active in each case. Thus the "degrees of freedom" for this new problem will be reduced from six to five. This reduction in freedom to the optimization procedure would be expected to reduce the final velocity of the missile at the terminal point.

The optimum parameters for the time-constrained solutions along with the optimal parameters for the minimum-time and the free-final-time problems are given in Table 4.11. The resulting final conditions for 


\section{TABLE 4.11}

Optimal Parameter Values for Time Constrained Trajectories

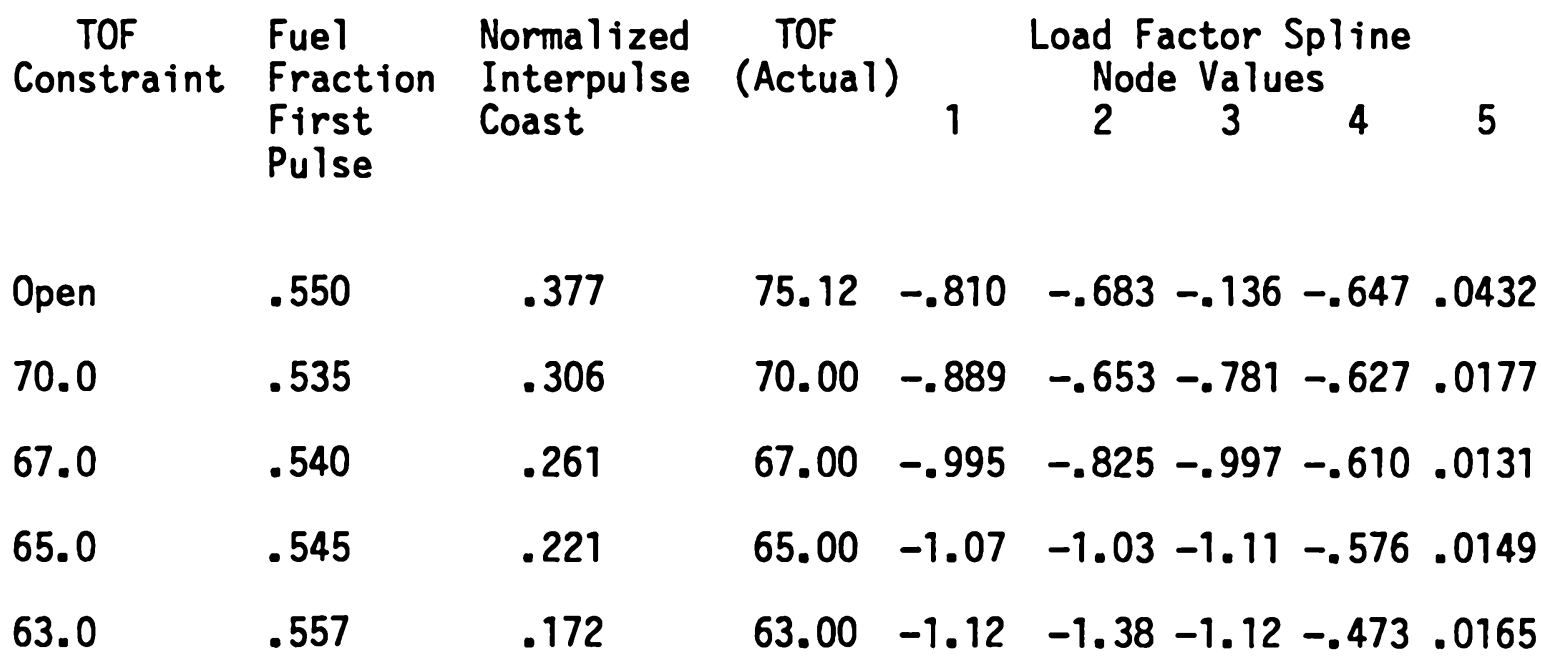

MINIMUM

TOF

TRAJECTORY $\quad .597$
.0741

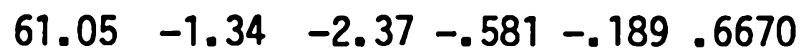


these same trajectories are given in Table 4.12. One of the most notable aspects of these results is that reducing the allowable time-of-flght constraint is lowered below 63.0 seconds. At that time constraint, the reduction in performance is only 6.0 percent compared to the free-final-time result. Figure 4.8 illustrates the tradeoff between terminal dynamic pressure with the mean ground speed. Like energy, the dynamic pressure shows a small variation of 10.2 percent decrease for a 20.9 percent increase in mean ground speed. As the final time constraint tightens, the total distance traveled to the target is reduced by decreasing the curvature of the trajectory. This is evidenced by the decreasing steepness of the final flight-path angle as the maximum time constraint is reduced. This pattern continues until the minimum-time-of-flight trajectory reaches the target with a positive flight-path angle. Because the curvature is reduced, the shorter trajectories have a greater portion on the flight at the lower altitudes. This coupled with the greater average speeds increases the energy losses due to aerodynamic drag. Achieving these shallower trajectories requires larger magnitude load factors early in the flight which also increases the aerodynamic drag losses.

As the final-time constraint tightens, the effect on the pulsing structure is not as great as might be expected. The fuel fraction allocated to the first pulse varies only over a small range and shows no definite trend. For the larger time constraints, the fuel fraction decreases as the time-of-flight decreases. But then as the constraint tightens still further it increases to a value greater than the 
TABLE 4.12

Time Constrained Trajectories Final Values

TOF

Constraint
Energy Mean Ground Speed

(feet) (feet/second)
Flight Path Angle (Radians)

\begin{tabular}{llll}
\hline Open & $167,000$. & 1600. & -.0406 \\
70.0 & $166,000$. & 1710. & -.0376 \\
67.0 & $163,000$. & 1790. & -.0347 \\
65.0 & $161,000$. & 1850. & -.0327 \\
63.0 & $157,000$. & 1900. & -.0246 \\
\hline
\end{tabular}

MINIMUM

TOF

TRAJECTORY

140,000

1970.

.123 


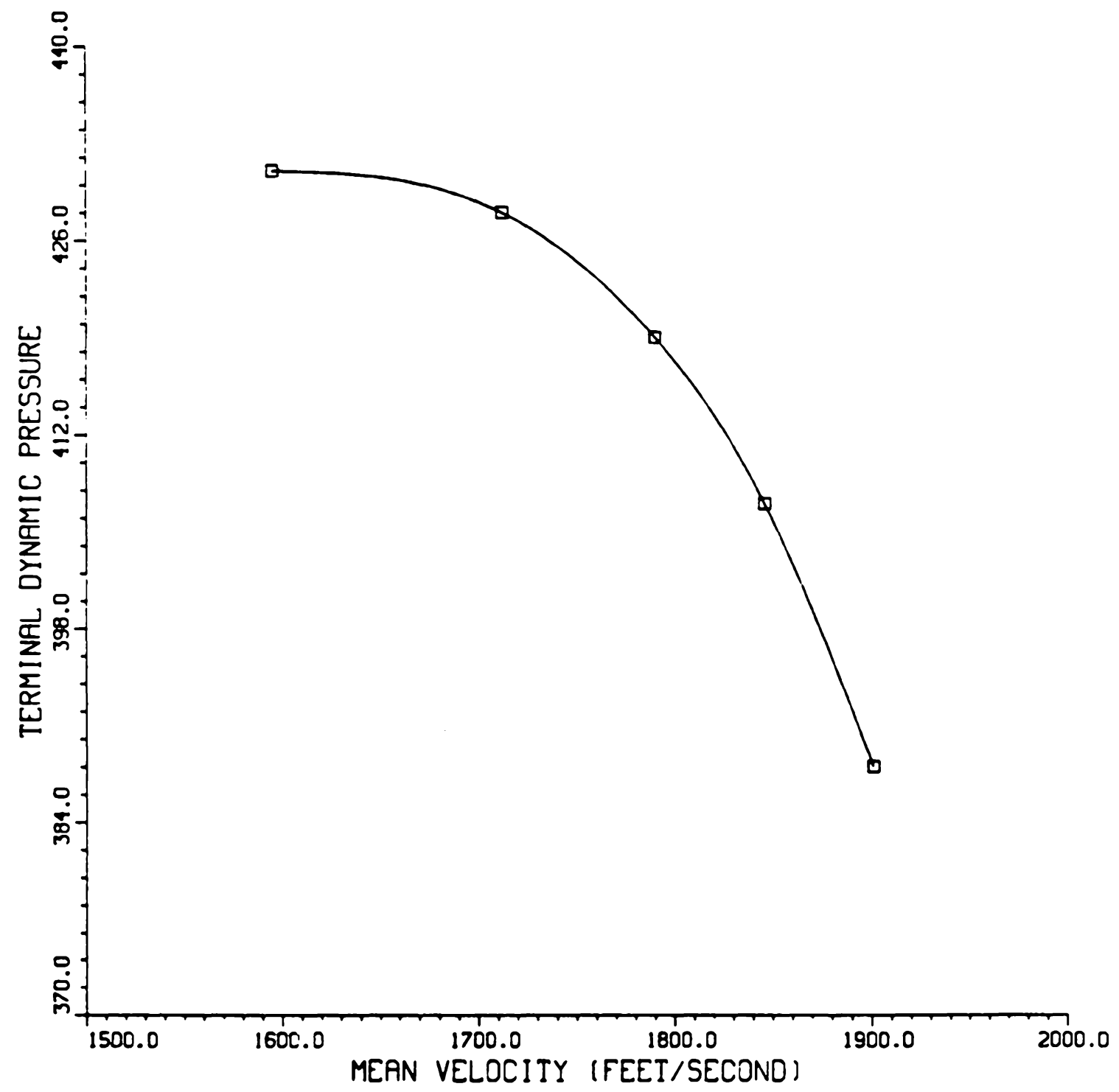

Figure 4.8 Effect of Time of Flight Constraint on Terminal Conditions 
free-time solution. For the larger time constraints, it is possible to easily boost the mean ground speed by decreasing the interpulse coast time. Dynamic pressure and drag are then reduced by burning more of the fuel on the second pulse. But as the constraint becomes tighter, it is necessary to also transfer fuel to the first pulse to boost the mean ground speed and still retain the best possible performance. The interpulse coast time varies with the decreasing time constraint as would be expected. For the shorter-duration flights, the coast time must be decreased until it reaches the coast time associated with the minimum-time-of-flight trajectory. 


\section{Chapter 5}

Conclusions

A number of problems in the optimization of the flight profiles and control histories of solid-fuel rockets were addressed. The first of these was the problem of continuously-variable thrust horizontal-lifting flight. The thrust history for optimizing the total boost-glide range of such a vehicle was determined by means of an optimal-control formulation by examination of the necessary conditions for an optimal control. The singular control developed for this problem was demonstrated to meet the sufficiency conditions for optimal control by Miele (2). Thus this control can be used as a benchmark against which the performance of approximations to the optimal-control methodology may be judged. The next portion of this study involved just such an approximate method to see how well it approximated the optimal-control solution.

The optimal-control formulation for the horizontal-fligit continuously-variable thrust boost-glide range maximization problen was approximated by a parameter-optimization methodology. In this approach, the mass-flow-rate time history was replaced by a set of three parameters. The first of these equals the fraction of fuel consumed in the initial maximum-mass-flow-rate burn. The second and third parameters represent nodal values of the single-segment linear spline used to represent the sustain arc of the optimal control. The mass-flow rate history determined for the parameter-optimization problem was found 
to closely approximate that obtained with the optimal-control formulation. The fuel fraction allocated to the initial maximum-mass-flow-rate segment was found to be within a mass-fraction value of .02 or about 6.0 percent of the optimal-control value. The mass-flow rates at the two sustain-arc nodes were not found to be in as close agreement with the corresponding mass-flow rate values on the sustain arc as given by the optimal-control formulation. The agreement was found to be within about 15.0 percent at the nodes. To determine the effect of increasing the fidelity of the approximation on the parameter-optimization solution, the problem was redone with a two-segment linear spline to model the sustain arc. The resulting parameter-optimization solution showed greater fidelity to the optimal-control solution. The error in the value of fuel-mass-fraction consumed in the initial maximum-mass-flow-rate arc decreased to 4.0 percent and the second half of the sustain spline approximated the sustain arc very closely. So it appears that the errors in the parameter-optimization solution arise from the approximations in the definition of the parameterization with respect to the optimal-control problem and as the parameterization is defined with greater fidelity the parameter-optimization solution should approach the optimal-control solution even more closely. So in a sense the use of the parameter-optimization methodology has been "validated". giving more confidence in its use to solve problems for which there is no corresponding analytical optimal-control solution. 
lith the confidence gained from the successful solution of the first problem, the next step was to do a problem of more practical interest for which there is no analytical optimal-control solution. The problem considered was that of determining the flight profiles which maximize the velocity at a specified final point for a pulsed solid-fuel missile with Mach-dependent aerodynamics. Both horizontal one-dimensional and elevation-plane two-dimensional trajectories were considered. Both two- and three-pulse motor configurations were optimized for the horizontal-flight problem. The results indicated that the use of a multi-pulse configuration offers substantial improvement sin the final velocity for this problem as compared to the boost-only type of motor. The two-pulse motor configuration offered a 163. percent increase while the three-pulse offered a 207. percent improvement. For both the two- and three-pulse configurations, it was found that the ignition of the second and third pulses occurred at approximately the same speed. This result tends to indicate that a criterion for the ignition of the non-boost pulses could be based on a relationship between dynamic pressure and the aerodynamic characteristics of the missile.

Calise (9) develops such a pulse-triggering algorithim by means of a reduced-order optimal-control method. Cne of the uses of parameter optimization is to "validate" the results of such reduced-order solutions. While the parameter-optimization method allows determination of the best pulse-ignition times and fuel allocation, the reduced-order method of (9) presents an algorithim for determining the best ignition 
time of a pulse for a fixed pulse size. This criterion states that the next pulse should be ignited if the following inequality is satisfied.

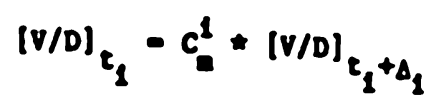

where the right hand side represents the velocity-to-drag ratio after the firing of the pulse multiplied by a varying mass correction term. This algorithim was run for the two-pulse motor configuration with the optimal fuel allocation as determined by the parameter-optimization method. It was found that the two methods exhibited exact agreement on the ignition times of the second pulse. Thus, this agreement tends to lend credence to both methodologies. Of course, the parameter-optimization method has the benefit of determining the optimal fuel allocation.

One step beyond this problem in complexity is that of a control which maximizes the final velocity at a specified final altitude and downrange for this same missile vertically launched. In addition to the motor parameters and the time of flight, five nodal values for the load-factor spline were optimized. The optimized trajectories for the one-pulse boost-only motor configuration and a two-pulse motor configuration were determined with and without time constraints. The final-time-free two-pulse problem revealed a motor pulsing structure unlike that for the one-dimensional problem with the second pulse being 
fired at approximately the mid-point of the trajectory. This early firing results from the gravity penalty associated with carrying propellant to higher potential energy states. The optimal tiyo-pulse trajectory exhibited greater time-of-flight and less curvature in its shape than the boost-only motor. Because of its lessened curvature and lower peak speeds, the two-pulse motor exhibits much less load-factor control activity which along with lower peak dynamic pressure allows for an 16.8 percent increase in terminal velocity as compared to the boost-only motor configuration. The optimal trajectories for both motors did not require load-factors which achieved the upper bound on the lift coefficient.

This result confirms the intuitive notion that it is necessary to avoid the high drag associated with high lift coefficients. The addition of minimum-mean-ground-speed constraints to the two-pulse motor configuration revealed a small latitude in speed control from the final-time-free trajectory to the minimum-time-of-flight trajectory. The boost-only configuration minimum-time-of-flight trajectory was also determined and was found to be longer than the t:yo-pulse trajectory, which used a very short delay between the pulses. The implication of these results is that the pulsed motor offers advantages in all aspects for trajectory shaping over the one-pulse motor.

The results of this study indicate that the parameter-optimization method is a viable method for the solution of a wide range of otpimal-trajectory problems for short-range, pulsed, solid-fuel missiles. It was found that for load-factor-history control schemes, 
the use of time as both the independent variable and the stopping criterion for the trajectory model was an effective procedure. In early runs range was used as a stopping criterion but the algorithim would sometimes encounter flight path-angles in excess of ninety degrees so that range was not necessarily monotone increasing. The combination of the parameter-optimization scheme with the trajectory model was able to effectively solve maximum-velocity, final-time-free and time-constrained problems as well as minimum-time-of-flight problems. 


\section{References}

1. Hibbs, A.R., "Optimum Burning Program for Horizontal Flight" Journal of the American Rocket Society. Vol. 22, July-August 1952. pp 206-212.

2. Meile, A. and Cicala, C.. "Generalized Theory of the Optimum Thrust Program for the Level Flight of Aircraft", Jet Propulsion, Vol. 26 No. 6. June, 1956, pp 442-456.

3. Leitmann, G., An Introduction to Optimal Control, McGraw-Hill Book Company, New York, 1966.

4. Keller, H.B., Numerical Solution of Two Point Boundary Value Problems, Society for Industrial and Applied Mathematics, Philadelphia, Pennsylvania, 1975.

5. We1l, K.H.. "Optimization of Tactical Aircraft Maneuvers Utilizing High Angles of Attack". AIAA Paper 80-1596. Danvers, Massachusetts, August, 1980.

6. Kelley, H.J.. "Method of Gradients", Optimization Techniques, G. Leitmann, Ed., Academic Press, New York, 1962.

7. Kelley, H.J. and Speyer, J.L., "Accelerated Gradient Projection", Lecture Notes in Mathematics 132. Springer-Verlag, Berlin, 1970.

8. Mummolo, F. and Lefton, L., "Cubic Spline and Cubic Spline Lattices for Digital Computation". Analytical Mechanics Associates, Inc. Report No. 72-12. August 1972, verson dated February, 1975.

9. Calise, A. and Nagy, J., "Necessary Conditions for Optimal Pulse Control". AIAA Paper No. 85-1955, Snowmass, Colorado, August, 1985.

10. Corlett. W.A.. "Supersonic Stability and Control Characteristics of a Cruciform Missile Model with Delta Wings and Aft Tail Fin Control", NASA TM 80171. 1979.

11. Hill, P. and Peterson, C.. Mechanics and Thermodynamics of Propulsion. Addision-Wes ley Publishing Company, Reading, Massachusetts, 1970. 
Appendix A

Missile Airframe Characteristics and Atmosphere Model

This section contains the airframe characteristics for the missile used in the pulsed-motor study described in Chapter 4. Also described is the atmospheric model used in the same study. The airframe characteristics consist of both the airframe aerodynamic properties and the mass properties of the missile. The missile used in thsi study is a single-stage solid-fuel planar wing anti-air missile with cruciform fins which was derived from the cruciform wing configuration described in (10). The aerodynamic characteristics consist of the trim drag coefficient as a function of Mach number and lift coefficient and the maximum lift coefficient as functgion of Mach number for the airframe at a roll angle of 45.0 degrees or in a cross configuration. The centerof-gravity location used for the trim calculations was a normalized value of 0.5078 from the nose tip, representing the burnout value. Also, for the maximum lift-coefficient values a maximum fin deflection of 40.0 degrees was used. Calculation of the trim drag coefficient is accomplished by means of a two-dimensional cubic spline in Mach number and lift coeeficient fitted through the drag data given in Table A.1. These data are derived from those given in (10) and have ben extendfed beyond the Mach-number range of 1.63 to 4.5 and the lift-coefficient range of 0.0 to 12.0 of the original source. The reasoning for this extrapolation is two-fold. The first is to provide aerodynamic data 
TABLE A. 1

Aerodynamic Drag Coefficients for Missile Airframe

Mach Number

$\begin{array}{lllllllllll}\mathrm{CL} & 0.0 & 1.69 & 2.1 & 2.5 & 2.86 & 3.95 & 4.63 & 5.0 & 6.0 & 10.0\end{array}$

$\begin{array}{rrrrrrrrrrr}0.0 & .59 & .60 & .62 & .60 & .57 & .45 & .40 & .38 & .37 & .37 \\ 1.0 & .60 & .65 & .65 & .65 & .65 & .65 & .76 & .79 & .78 & .78 \\ 2.0 & .70 & .80 & .80 & .82 & .90 & 1.1 & 1.25 & 1.3 & 1.31 & 1.32 \\ 3.0 & .90 & 1.00 & 1.15 & 1.33 & 1.43 & 1.65 & 1.76 & 1.79 & 1.80 & 1.80 \\ 4.0 & 1.20 & 1.30 & 1.55 & 1.82 & 2.08 & 2.15 & 2.30 & 2.35 & 2.36 & 2.36 \\ 5.0 & 1.70 & 1.75 & 2.00 & 2.32 & 2.50 & 2.68 & 2.85 & 2.90 & 2.92 & 2.92 \\ 6.0 & 2.20 & 2.35 & 2.50 & 2.90 & 3.20 & 3.40 & 3.42 & 3.43 & 3.43 & 3.43 \\ 100 & 50.00 & 54.00 & 55.00 & 56.00 & 70.00 & 60.00 & 55.00 & 54.00 & 54.00 & 54.00\end{array}$


for the lower Mach numbers near the beginning and end of the trajectory. The second reason is to extend the splines to values of the Mach number and of the lift coefficient beyond those to be encounrtered during operation of the trajectory model. This ensures well-conditioned behavior of the optimizer/trajectory-model combination. The maximum lift-coefficient as a function of Mach number is presented in Table A.2 and was derived from (10). Representation of the maximum liftcoefficient is by a one-dimensional cubic spline with Mach number as the independent variable and fit through the data in the table. The aerodynamic reference area for the aerodynamic data in these tables is the body cross-sectional area of 0.545 square feet.

The remaining information required to define the missile is the weight and propulsion properties. These values were determined by means of the author's experience and from open sources of information on missile subsystem weight properties and are typical of modern surface-to-air missiles with aluminum and steel structures. The weight breakdown for the missile is given in Table A.3. The missile propulsion properties given in Table A.4 are for a 220. second specific impulse solid fuel rocket motor and were obtained from (11). It should be noted that no weight penalty has been associated with the pulsing of the engine.

The trajectory model requires the definition of a model of the atmosphere. In the present study, local sonic velocity and atmospheric denisty are computed by use of cubic splines with altitude as the 


\section{TABLE A.2}

\section{Maximum Airframe Lift Coefficients}

Mach Number

0.0

1.6

2.1

2.86

3.95

4.63

6.0

7.0
Maximum Lift Coefficient

6.0

6.0

6.4

4.1

3.75

3.2

3.1

3.1 
TABLE A.3

Missile Weight Breakdown

Subsystem

Airframe

Guidance

Warhead

Fuel

Launch Weight
Weight (pounds)

405.0

80.0

60.0

431.0

976.0 


\section{TABLE A.4}

Missile Propulsion Characteristics

Fuel Type

Exhaust Velocity

Exhaust Area

Exhaust Pressure
JPN! Ballistic

7100.0 feet/second

0.545 square feet

2116.0 pounds/square foot 
independent variable. These are fit through the natural log of the density and the sonic velocity as a function of the altitude normalized by a scaling factor of 10000.0 feet. These data are presented in Table A. 5. 
TABLE A.5

Atmospheric Model
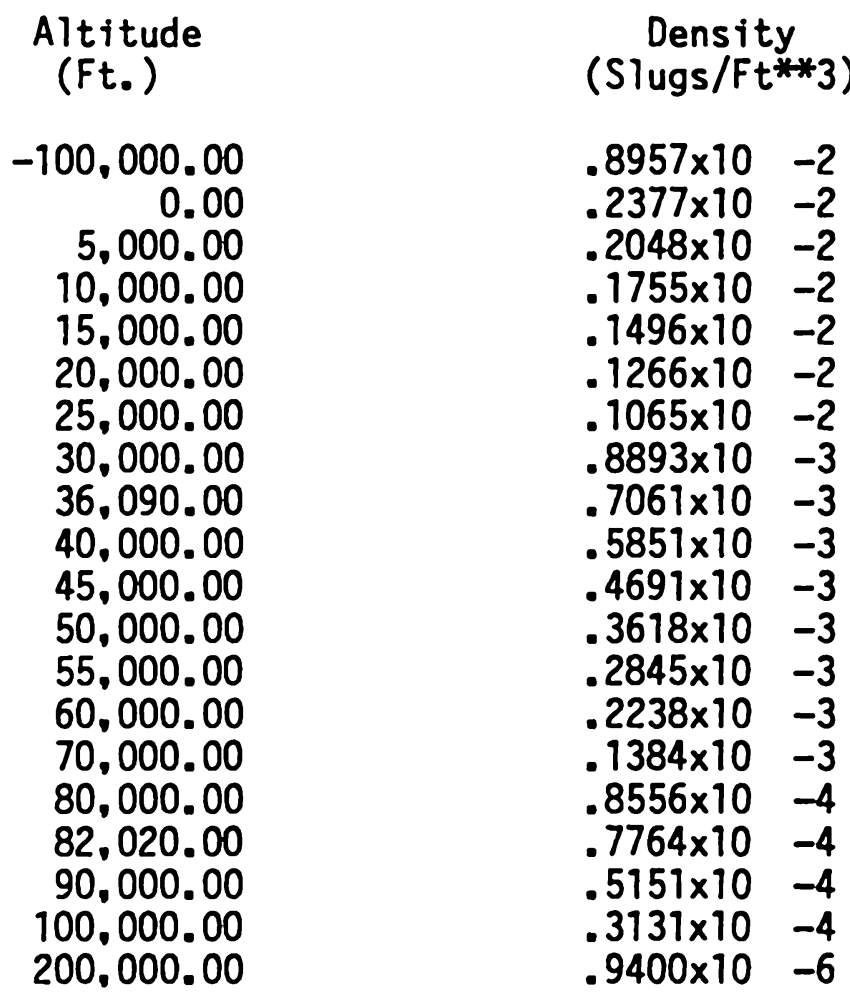

Speed of Sound

(Ft/Sec)

1496.00

1116.00

1097.00

1077.00

1057.00

1037.00

1016.00

994.70

972.00

968.10

968.10

968.10

968.10

968.10

968.10

968.10

968.10

984.20

1004.00

1056.40 
The vita has been removed from the scanned document 